Int. J. Mol. Sci. 2002, 3, 604-638

International Journal of

Molecular Sciences

ISSN 1422-0067

www.mdpi.org/ijms/

\title{
Implicit and Explicit Coverage of Multi-reference Effects by Density Functional Theory
}

\author{
Dieter Cremer $^{1 \star}$, Michael Filatov ${ }^{1}$, Victor Polo $^{1}$, Elfi Kraka ${ }^{1}$ and Sason Shaik ${ }^{2}$ \\ ${ }^{1}$ Department of Theoretical Chemistry, Göteborg University, Reutersgatan 2, S-413 20 Göteborg, Sweden \\ *Electronic-mail: Cremer@theoc.gu.se \\ ${ }^{2}$ Department of Organic Chemistry and The Lise Meitner-Minerva Center for Computational Quantum Chemistry, \\ Hebrew University, Jerusalem, 91904 Jerusalem, Israel
}

Received: 4 December 2001 / Accepted: 6 April 2002 / Published: 30 June 2002

\begin{abstract}
Multi-reference effects can be covered by density functional theory (DFT) either implicitly via the exchange-correlation functional or explicitly via the form of the Kohn-Sham wave function. With the help of the exchange hole it is shown that the self-interaction error of the exchange functional will mimic long-range electron correlation effects if restricted Kohn-Sham theory is used. Functionals based on Slater or Becke exchange have a relatively large self-interaction error and, therefore, lead to a relatively large implicit coverage of long-range correlation, which, because of the possibility of doublecounting of electron correlation, has to be considered when using these functionals in connection with two- or multi-configurational descriptions based on ensemble DFT methods such as REKS (spinRestricted Ensemble-referenced $K S$-DFT). Arguments are given that a REKS description of a multireference problem avoids a double-counting of long-range correlation effects, in particular as in this situation the self-interaction error of the exchange functional simulates more short- rather than longrange correlation effects. There is, however, no guarantee that the short-range effects are not doublecounted, namely once via the exchange and once via the correlation functional. Therefore, one should use hybrid functionals such as B3LYP in connection with multi-reference DFT methods because for hybrid functionals the self-interaction error and by this the implicit coverage of long(short)-range correlation effects is reduced due to the admixture of exact exchange. This rule applies also to broken-symmetry UDFT, which performs better with hybrid rather than GGA functionals. A way of avoiding the implicit coverage of multi-reference effects is given by the combination of wave function theory and DFT methods. The advantages and disadvantages of CAS-DFT are discussed and it is shown that an effective reduction of a double-counting of correlation effects is possible within this method.
\end{abstract}

Keywords: Exchange functional, self-interaction error, non-dynamic electron correlation, multireference effects, two-configurational DFT, REKS, ensemble theory, CAS-DFT

(c)2002 by MDPI, Basel, Switzerland. Reproduction for noncommercial purposes permitted. 


\section{Introduction}

It is generally assumed that standard Kohn-Sham density functional theory (DFT) [1-4] carried out with approximate exchange-correlation functionals (approximate DFT) as they are currently in use covers beside exchange correlation effects just short-range Coulomb correlation (dynamic electron correlation). Nevertheless, approximate Kohn-Sham DFT performs astonishingly well for electronic systems that have been identified by wave function theory (WFT) to represent notorious multi-reference problems. Of course, this observation has to be qualified in a number of ways: a) One cannot expect approximate KS-DFT to be always successful in the case of multi-reference systems. Dramatic failures are also known. [3-8] b) One has to specify whether a satisfactory performance is obtained by the restricted, unrestricted or broken-symmetry unrestricted version of DFT: RDFT, UDFT or BSUDFT. Multi-reference systems can often be better described by BS-UDFT rather than RDFT or UDFT. [9] c) The performance of DFT in the case of a multi-reference system depends on the choice of the exchange-correlation functional. Local density approximation (LDA) functionals perform significantly different from GGA (generalized gradient approximation) or hybrid functionals in the case of multi-reference systems. [2-4]

The essence of KS-theory is that if the exact exchange-correlation functional would be known (leading to exact KS DFT), any electronic system, whether multi-reference or single-reference in nature could be accurately described. [10] Obviously, it is the approximate nature of the current exchange-correlation functionals which leads to the varying performance of KS-DFT in the case of multi-reference problems. In this work, we will critically discuss the question to which extent DFT can be used to reliably describe molecules with distinct multi-reference character. We will show that approximate DFT already incorporates some multi-reference effects via the form of its exchange functional [11-13] and that this is inherently connected to the self-interaction error, [14-16] from which all current exchange functionals suffer. [14-26] This will be referred to as implicit coverage of multi-reference effects, which has serious implications for the explicit account of multi-reference effects via an extension of standard KS-DFT. For the explicit account of multi-reference effects, DFT offers today two major routes, namely a) the extension of standard DFT to ensemble DFT [27-30] and b) the merging of DFT with techniques used in WFT. The latter possibility includes methods such as two-configurational DFT (GVB-DFT) [31] and multi-configurational DFT such as CAS-DFT. [32] The former route is realized in REKS(spin-Restricted Ensemble-referenced $K S$ )-DFT. Both approaches will be discussed where the focus of the discussion will be on the principal question on how multi-reference systems can be described in the best way using these methods.

If one discusses multi-reference effects, one should specify what effects are actually addressed by this term. According to a general scheme of classifying multi-reference systems suggested by Gräfenstein and Cremer [9,32] one can distinguish between a) closed shell and high-spin open-shell systems that are satisfactorily described by a single configurational state function (CSF) represented by a single determinant (type 0 systems), b) low-spin open-shell systems such as singlet biradicals that require two- or multi-determinantal descriptions where these determinants form still one CSF (type I systems), genuine two- and multi-configurational systems where each $\mathrm{CSF}$ can be represented by a single determinant (type II systems such as ozone $\left({ }^{1} A_{1}\right)$, $\mathrm{C}_{2}\left({ }^{1} \Sigma_{g}^{+}\right)$, dissociating A-A molecules, etc.), and finally systems with multi-determinantal, multi-configurational wave functions (type III systems such as the Myer's biradical). [9] The current article will concentrate on the correct description of type II and type III systems because this implies the inclusion of long-range Coulomb correlation effects (non-dynamic or static electron correlation) into DFT. It will not consider type I systems although their correct description is already problematic at the DFT level and requires methods such as ROSS [33] or ROKS [34]. However, type I 
systems do not possess (by definition) any long-range correlation and therefore are not considered to represent multi-reference problems.

The objectives of this work are threefold: a) First, we will clarify what types of correlation effects are introduced by approximate exchange functionals under different conditions, i.e. RDFT, UDFT or BS-UDFT. In this connection, difference densities and representations of the exchange hole will be used to support the discussion. b) Then, we will test the performance of LDA, GGA, and hybrid functionals in combination with two-configurational DFT in the case of singlet biradicals with significant multireference character. For this purpose, a new program was developed, which can carry out energy calculations and geometry optimizations with hybrid functionals. c) Implicit and explicit coverage of multireference effects is analyzed for different situations and the possibility of double-counting of electron correlation effects in multi-configurational DFT is discussed.

Results of this work are presented in the following way. In section 2, we will analyze the implicit coverage of multi-reference effects by DFT. In the following sections, explicit coverage of multi-reference effects either by ensemble DFT (sections 3 and 4) or WFT-DFT methods (section 5) will be discussed. Conclusions are summarized in section 6 .

\section{Implicit Account of Multi-reference Effects by Standard DFT}

The exchange-correlation hole $h_{X C}$ is defined by Eq. (1) (see, e.g., Ref. 2):

$$
h_{\mathrm{XC}}\left(\mathbf{r}_{1}, \mathbf{r}_{2}\right)=\frac{P\left(\mathbf{r}_{1}, \mathbf{r}_{2}\right)}{\varrho\left(\mathbf{r}_{1}\right)}-\varrho\left(\mathbf{r}_{2}\right)
$$

where $P\left(\mathbf{r}_{1}, \mathbf{r}_{2}\right)$ is the pair density distribution, $\rho(\mathbf{r})$ is the one-electron density distribution, $\mathbf{r}_{1}$ denotes the position of the first electron (reference electron), and $\mathbf{r}_{2}$ the position of the second electron. The exchange-correlation hole can be split up into exchange hole $h_{X}$ and correlation hole $h_{C}$, which are related to exchange and Coulomb correlation, respectively. The exchange hole is negative or equal to zero everywhere and fulfills the sum rule [2]:

$$
\begin{gathered}
h_{X}\left(\mathbf{r}_{1}, \mathbf{r}_{2}\right) \leq 0 \\
\int \mathrm{d}^{3} r_{1} \mathrm{~d}^{3} r_{2} h_{X}\left(\mathbf{r}_{1}, \mathbf{r}_{2}\right)=-1 .
\end{gathered}
$$

Exchange decreases the probability of finding two electrons with the same spin at the same position to zero, however does not affect the corresponding probability for opposite-spin electrons. For a closed shell system, the HF exchange hole is exact. It reflects the electronic structure of the molecule provided the reference electron is positioned in the bonding or lone-pair region. In the first case, the HF exchange hole is delocalized over the bond region and the regions of the bonded atoms (reflecting the form of the bond orbital) while in the second case its structure can be deduced from the form of the lone-pair orbital and the overlap of the latter with other orbitals. A deeper exchange hole corresponds to a more negative exchange energy.

The structure of the exchange hole is influenced by two factors, namely a) the self-exchange of the electrons (intraelectronic exchange) and b) the Fermi exchange correlation between different electrons possessing the same spin. The intraelectronic part of the HF exchange hole fulfills Eq.s (2) and (3) while the interelectronic exchange hole can be both positive and negative and sums to zero. The former takes care of the self-interaction problem in the way that the corresponding self-exchange energy exactly annihilates the self-repulsion energy of an electron 
with itself. This however is not true in the case of the DFT exchange hole, which leads to the self-interaction error (SIE) of DFT. Approximate DFT, contrary to HF, does not fulfill Eq.s (4) and (5)

$$
\begin{gathered}
E_{J}[\varrho]+E_{X}\left[\varrho_{\alpha}, 0\right]=0 \\
E_{C}\left[\varrho_{\alpha}, 0\right]=0
\end{gathered}
$$

which require that for the ground-state density of a system containing just one $\alpha$-spin electron, i.e. $\int \varrho_{\alpha}(\mathbf{r}) d \mathbf{r}=1$, and $\varrho_{\beta}(\mathbf{r})=0$, the self-exchange energy $E_{X}\left[\varrho_{\alpha}, 0\right]$ exactly cancels the self-repulsion $E_{J}[\varrho]$ of the electron and that there is no correlation of an electron with itself. All commonly used approximate exchange functionals violate condition (3) and lead to a relatively large exchange SIE. The SIE of approximate correlation functionals is smaller and even zero in the case of those correlation functionals, which were derived from WFT methods as for example the LYP functional. Therefore, we will consider in the following just the exchange SIE.

While the HF exchange hole is often delocalized, the DFT exchange hole is always localized and concentrated at the position of the reference electron (LDA exchange holes) or in the vicinity of the reference electron (GGA exchange holes). One can consider the differences between HF and DFT exchange hole in two ways:

a) The SIE converts the delocalized HF exchange hole into a localized DFT exchange hole. Although it is an error by nature, it introduces in this way some useful electron correlation effects (to be discussed in the following), which significantly influence the performance of DFT. Self-interaction corrected DFT (SIC-DFT) annihilates the SIE and in this way also destroys the electron correlation effects mimicked by the SIE. Accordingly, SIC-DFT is closer to HF as directly reflected by the exchange holes generated by the two methods.

b) The LDA and GGA exchange approximations are constructed to genuinely describe both exchange and leftright correlation. The self-interaction correction brings the DFT model closer to HF and destroys the left-right correlation LDA (or GGA) has built in (see, e.g. Ref. 12a).

Reasoning a) partitions the correlation effects covered by the exchange-correlation functional to have a better basis for analyzing these effects in detail and to have a basis for developing better exchange-correlation functionals. Along this line, the necessity of describing first exchange correctly as the larger and more important effect is emphasized and in so far the conversion of the delocalized HF into a localized DFT exchange hole is considered as an error. Reasoning b) is based on the original DFT strategy, namely all electron interaction effects difficult to describe are contracted in one functional, which, if correctly parametrisized, leads to the true energy. Only in those cases where this approach seriously fails, one has to use methods such as SIC-DFT, which describe the exchange interactions of the electrons more correctly.

In this work, we follow reasoning a) because we are interested to analyze how various correlation effects are covered by the exchange-correlation functional. Therefore, we emphasize the SIE of DFT although it is clear that the latter may be considered as wanted or needed. This becomes obvious by defining the SIE as the difference DFT - SIC/DFT rather than the difference SIC/DFT - DFT as one should do if one speaks of an error. However, the former alternative is preferable because the SIE is related to an electron correlation effect thus suggesting the following correspondence:

$$
\begin{gathered}
E(\text { true })=E(H F)+E(\text { corr }) \\
E(D F T, X-\text { only })=E(S I C / D F T, X-\text { only })+E(S I E, X-\text { only })
\end{gathered}
$$

and

$$
E(\text { corr })=E(\text { true })-E(H F)
$$




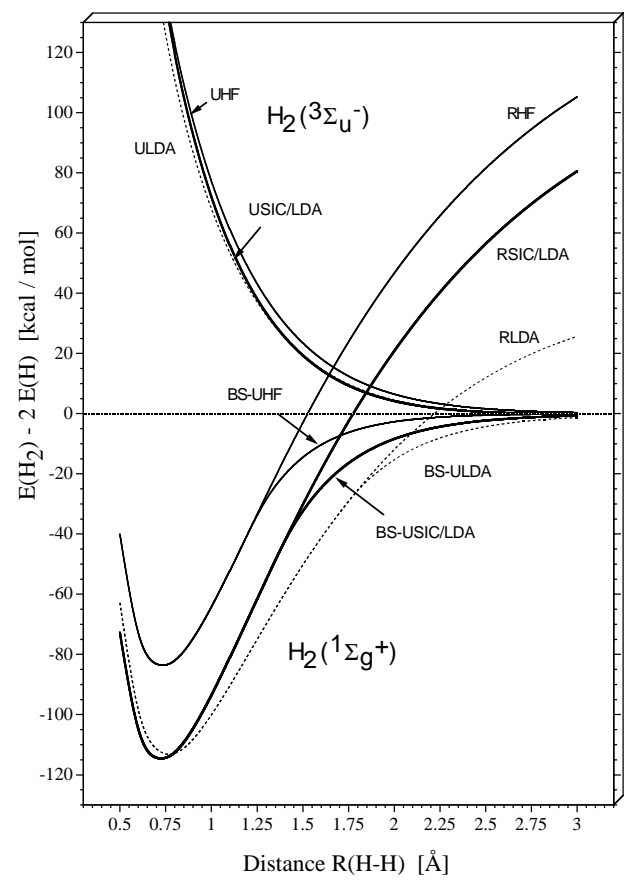

Figure 1: Potential curves of the ${ }^{1} \Sigma_{g}^{+}$ground state and the ${ }^{3} \Sigma_{u}^{-}$excited state of $\mathrm{H}_{2}$ calculated with a cc-pVTZ basis set at various levels of theory.

$$
E(S I E)=E(D F T)-E(S I C / D F T)
$$

The DFT energy E(DFT,X-only) obtained from an exchange-only calculation is considered as a correlationcorrected energy approximating the true energy $\mathrm{E}$ (true), which is the sum of the HF energy $\mathrm{E}$ (HF) (corresponding to $\mathrm{E}(\mathrm{SIC}-\mathrm{DFT}, \mathrm{X}$-only)) and the correct correlation energy $\mathrm{E}$ (corr) where the latter term is approximated by the self-interaction energy $\mathrm{E}(\mathrm{SIE}, \mathrm{X}$-only)) in an exchange-only DFT calculation. It is of course still the question which correlation effects are included into DFT via the SIE of the exchange functional.

Figure 1 shows the potential curves for $\mathrm{H}_{2}$ in its ${ }^{1} \Sigma_{g}^{+}$ground state and its ${ }^{3} \Sigma_{u}^{-}$excited state as calculated at different levels of theory with Dunning's cc-pVTZ basis set [35]: RHF (restricted Hartree-Fock), UHF (unrestricted Hartree-Fock), and BS(broken-symmetry)-UHF, RLDA, UDLA, and BS-ULDA, RSIC/LDA, USIC/LDA, and BSUSIC/LDA where for the exchange-correlation functional SVWN5 was used (S: Slater exchange [36]; VWN5: VoskoWilk-Nusair correlation [37]). The self-interaction corrected DFT (SIC-DFT) was obtained using the method of Perdew and Zunger [17,18] in a self-consistent-field version. [19-22,38]

Figure 2 shows HF and DFT exchange holes in a one-dimensional representation along the internuclear axis for the dissociating $\mathrm{H}_{2}\left({ }^{1} \Sigma_{g}^{+}\right)$molecule at a distance of 1.5 (Figures $2 \mathrm{a}$ and $2 \mathrm{~b}$ ) and $3.0 \AA$ (Figures $2 \mathrm{c}$ and $2 \mathrm{~d}$ ) between 
a)

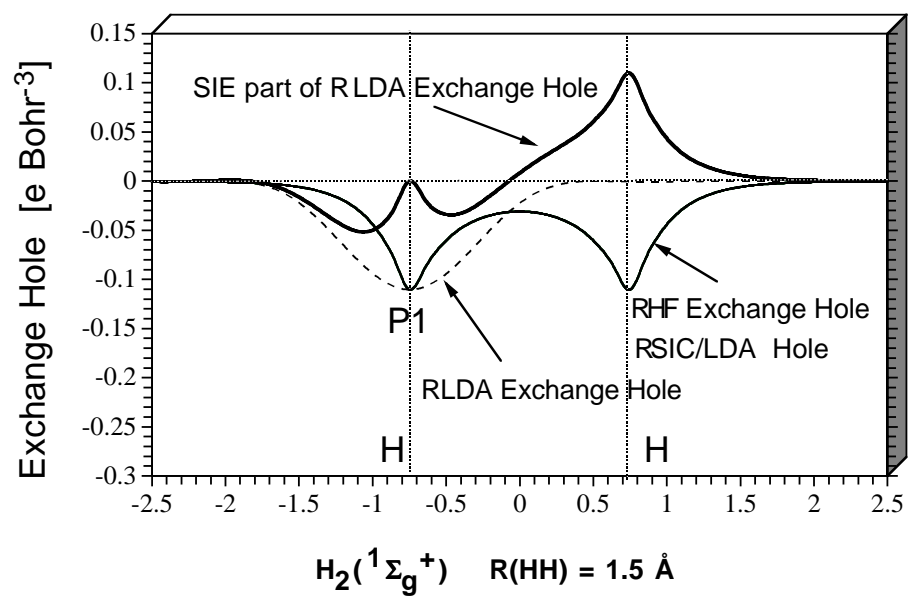

b)

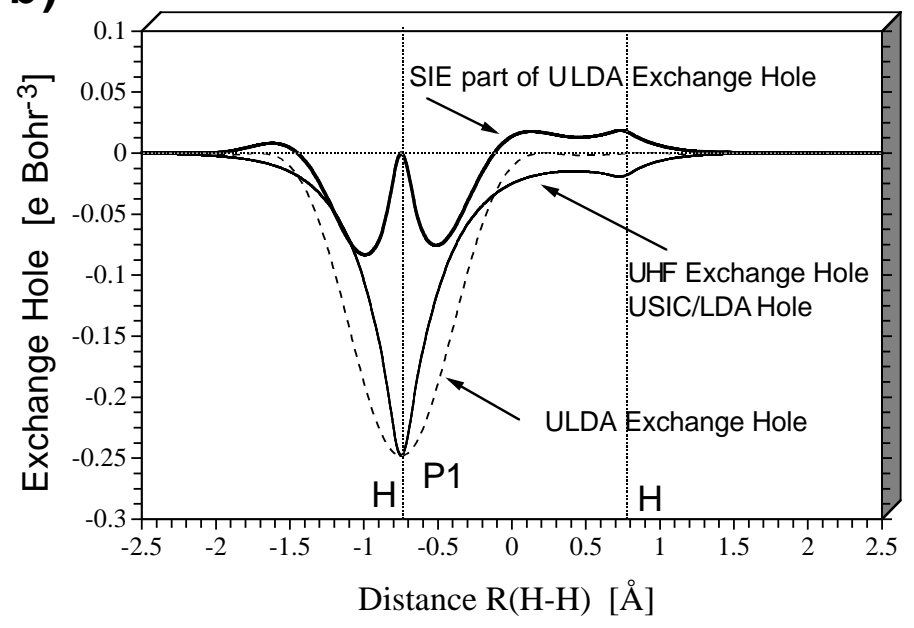

Figure 2: Parts a and b. For parts c and d and caption, see next page. 
c)

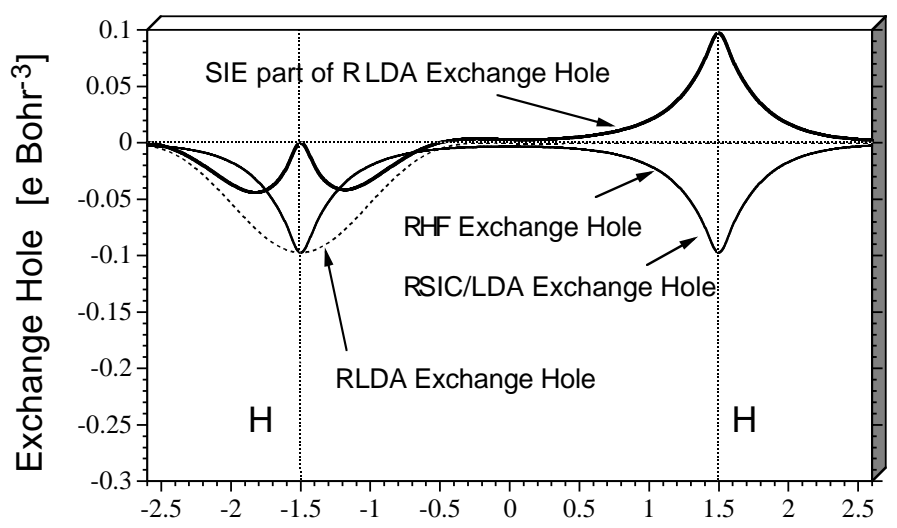

d)

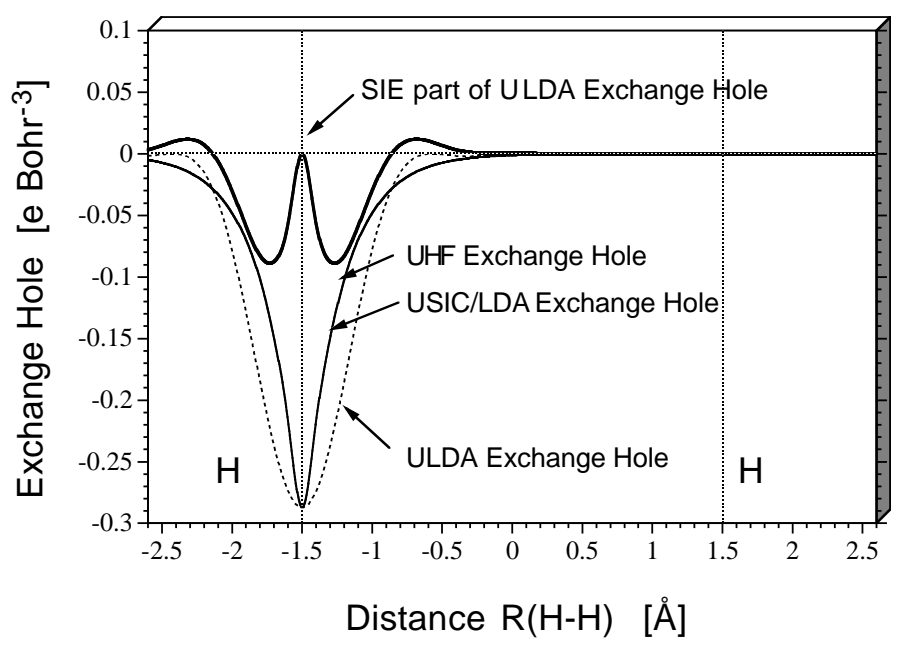

Figure 2: Graphical representation of the exchange hole calculated for the dissociating $H_{2}\left({ }^{1} \Sigma_{g}^{+}\right)$molecule along the bond axis for the situation that the reference electron is at the left $\mathrm{H}$ nucleus (position P1). The SIE part of the LDA exchange hole is given by the line in bold print. a) $\mathrm{R}(\mathrm{H}-\mathrm{H})=1.5 \AA$ : RHF, RSIC/LDA (both the normal line), and RLDA (dashed line) calculations. b) R(H-H) =1.5 A: BS-UHF, BS-USIC/LDA (both the normal line), and BS-ULDA calculations. c) R(H-H) $=3.0 \AA$ : RHF, RSIC/LDA (both the normal line), and RLDA (dashed line) calculations. d) $\mathrm{R}(\mathrm{H}-\mathrm{H})=3.0 \AA$ : BS-UHF, BS-USIC/LDA (both the normal line), and BS-ULDA calculations. All calculations with a cc-pVTZ basis set using in all cases the HF density to avoid differences because of differences in the density. 
the nuclei as obtained with a restricted description (Figures 2a and 2c) and an unrestricted description (Figures 2b and $2 \mathrm{~d}$ ). The exchange holes were calculated with the same density (taken from the HF calculations) to simplify the comparison so that just differences in the electron correlation effects covered by HF and DFT, respectively, need to be discussed. In Figures $2 \mathrm{a}$ to $2 \mathrm{~d}$, the exchange hole functions relate to the probability of finding the second electron when the reference electron is positioned at the left $\mathrm{H}$ nucleus.

Restricted theory is not able to describe dissociation correctly, which has been amply discussed and analyzed during the past 5 decades. [39] Unrestricted theory in its broken-symmetry form qualitatively corrects restricted theory by mixing the first doubly excited state with the ground state configuration thus lending HF some twoconfigurational character at the expense of spin contamination and the inclusion of higher spin states. [9] Close to the equilibrium geometry of $\mathrm{H}_{2}\left({ }^{1} \Sigma_{g}^{+}\right)$, the two electrons are equally shared by the two $\mathrm{H}$ atoms thus establishing the $\mathrm{H}-\mathrm{H}$ bond. In this situation, the only Coulomb correlation effects are of the short-range, dynamic nature. HF does not cover any dynamic electron correlation effects and, therefore, it underestimates the strength of the $\mathrm{HH}$ bond (Figure 1) as documented by a dissociation energy $D_{e}$ of $83.7 \mathrm{kcal} / \mathrm{mol}$ (calculated relative to twice the UHF energy of $\left.H\left({ }^{2} S\right)\right)$.

The LDA description at the equilibrium geometry leads to a more stable $\mathrm{HH}$ bond $\left(D_{e}=113.3 \mathrm{kcal} / \mathrm{mol}\right)$ where this difference is caused by a) the VWN5 correlation functional (major effect) and b) the self-interaction error of the Slater exchange functional (minor effect). [14-16] The two effects can be separated carrying out a SIC-LDA calculation, again using the HF density. The SIC-LDA calculation leads to the same exchange energy as the HF calculation and since the same correlation functional is used, [40] the difference between LDA and SICLDA (Figure 1) reflects the consequences of the self-interaction error of Slater exchange. Although differences are relatively small, the self-interaction error causes a) the $\mathrm{HH}$ bond length to increase from $0.727 \AA$ (SIC-LDA) to $0.766 \AA$ (LDA) and b) the $D_{e}$ value to decrease from 114.7 to $113.3 \mathrm{kcal} / \mathrm{mol}$ (Figure 1 ).

If $\mathrm{H}_{2}\left({ }^{1} \Sigma_{g}^{+}\right)$dissociates, the error caused by the lack of dynamic electron correlation will decrease slightly from its relatively large value of $31 \mathrm{kcal} / \mathrm{mol}$ at the equilibrium distance to about $25 \mathrm{kcal} / \mathrm{mol}$ at $3 \AA$ as is documented by the RHF and the RSIC-LDA dissociation curves (Figure 1). The self-interaction error given by the difference RLDA - RSIC/LDA changes in a different way: With increasing distance $\mathrm{R}(\mathrm{H}-\mathrm{H})$, it rapidly increases from 0 $(\mathrm{R}(\mathrm{H}-\mathrm{H})=0.766 \AA)$ to about $55 \mathrm{kcal} / \mathrm{mol}(3 \AA)$. However, the self-interaction error of the unrestricted description given by the difference BS-ULDA - BS-USIC/LDA decreases beyond the bifurcation point (SIC/LDA: 1.363; LDA: $1.745 \AA$, Figure 1) rapidly to zero for increasing $\mathrm{R}(\mathrm{H}-\mathrm{H})$.

These relationships can be easily explained based on the known properties of restricted and unrestricted theory as well as the structure of the exchange holes given by HF or DFT (Figure 2). Restricted theory erroneously describes cleavage of the $\mathrm{HH}$ bond partially homolytic, partially heterolytic. Dynamic electron correlation decreases with increasing $\mathrm{R}(\mathrm{H}-\mathrm{H})$ because of the expansion of the doubly occupied $\sigma_{g}$ orbital. - The consequences of the selfinteraction error of the exchange functional can be assessed by inspection of Figure 2. Exact RHF exchange implies a delocalized exchange hole with deep pockets at the positions of the nuclei and a shallow (close to zero) depression in the internuclear region (Figure 2a). Since, for $\mathrm{H}_{2}\left({ }^{1} \Sigma_{g}^{+}\right)$or any other spin-coupled 2-electron system, exchange is equal to the self-exchange (intra-electronic exchange), the exchange hole is static (independent of the position of the reference electron) and equal to $-1 / 2$ the electron density distribution $\rho(\mathbf{r})$. When SIC-LDA is calculated with the same density, it leads to exactly the same delocalized, static exchange hole. In this way, the form of both the HF and the SIC-LDA exchange hole provides electronic structure information.

The LDA exchange hole itself is localized rather than delocalized, depends on the position of the reference 
electron, and adopts a spherically symmetric form. At its deepest point it is equal to $-1 / 2 \rho(\mathbf{r})$, but otherwise it does not contain any electronic structure information. Since the sum rule implies that both LDA and HF exchange hole integrate to -1, a deep LDA exchange hole is narrow while a shallow LDA exchange hole is much more diffuse.

Clearly, the enforced spherically symmetric form of the LDA exchange hole can only be obtained from the correct SIC-LDA delocalized exchange hole by adding the hole due to the self-interaction error. The self-exchange hole of LDA is obtained by subtracting the SIC-LDA from the LDA exchange hole (Figure 2). It shows that for the reference electron being at the left $\mathrm{H}$ nucleus, the self-interaction error drastically increases the probability of finding the second electron at the right $\mathrm{H}$ nucleus. Hence, it leads to left-right long-range electron correlation and explains why the RLDA description apart from the fact that it covers dynamic electron correlation (difference RHF - RSIC/LDA) does not suffer so much from the basic failure of restricted theory when describing bond dissociation.

Based on Figure 2 the following conclusions can be drawn.

1) With increasing distance $\mathrm{R}(\mathrm{H}-\mathrm{H})$, the exchange hole becomes more and more delocalized. Accordingly the self-interaction error of the LDA exchange functional increases in magnitude and mimics more strongly left-right correlation.

2) As a consequence of 1), the RLDA solution becomes more stable than the RHF solution for the dissociating $\mathrm{H}_{2}\left({ }^{1} \Sigma_{g}^{+}\right)$molecule. The bifurcation point, at which RLDA becomes unstable relative to ULDA is shifted to a larger $\mathrm{R}(\mathrm{H}-\mathrm{H})$ value (from 1.212 to $1.745 \AA$, Figure 1 ). The more long-range correlation effects are covered by a given method, the more stable it becomes.

3) The energy difference between RLDA and RSIC/LDA is increased due to the increase of the self-interaction error.

Broken-symmetry unrestricted theory corrects restricted theory by the mixing of HOMO and LUMO thereby leading to an effective separation of the bonding electrons of the dissociating $\mathrm{H}_{2}$ molecule. Homolytic dissociation is qualitatively described in the correct way. This is also reflected by the exchange hole of unrestricted theory (Figure 2b). The BS-UHF exchange hole is localized at the position of the reference electron (taken to be at the left nucleus, Figure 2) indicating that there is a low probability of finding the second electron at the left $\mathrm{H}$ atom. Hence the situation of an isolated $\mathrm{H}$ atom with one electron is approached as required by homolytic dissociation. The LDA exchange hole, which is actually optimized for the situation of the isolated atom, now provides a more accurate description of exchange than it could do in the case of $\mathrm{H}_{2}\left({ }^{1} \Sigma_{g}^{+}\right)$at its equilibrium geometry. SIC-LDA and LDA exchange hole do no longer differ significantly, and their difference mimics more short-range rather than long-range electron correlation. Accordingly, the self-interaction error is rather small.

4) The explicit two-configurational description of long-range electron correlation as introduced by the BS-UDFT solution reduces the self-interaction error for increasing distance $\mathrm{R}(\mathrm{H}-\mathrm{H})$ to a small value, i.e. the self-interaction error for the isolated $\mathrm{H}$ atoms is close to zero. This is reflected by the difference BS-ULDA - BS-USIC/LDA (Figure $1)$.

5) With increasing $\mathrm{R}(\mathrm{H}-\mathrm{H})$, the self-interaction error mimics more short-range rather than long-range electron correlation. In the region of spin-recoupling from a closed-shell to an open-shell singlet, both long-range and short-range correlation effects are simulated by the exchange functional.

In the triplet state, both the UHF and ULDA exchange hole are localized and, consequently, the self-interaction error is small. USIC/LDA and ULDA hardly differ while however remaining differences become slightly larger for smaller $\mathrm{R}(\mathrm{H}-\mathrm{H})$ values (Figure 1$)$. The self-interaction error simulates small amounts of dynamic rather than non-dynamic electron correlation. Hence, one can focus primarily on the description of the singlet state when 
analyzing singlet-triplet splittings as described by various DFT approaches.

The relationships demonstrated here for $\mathrm{H}_{2}\left({ }^{1} \Sigma_{g}^{+}\right)$in the case of a LDA functional are also true for other multireference systems. As a second example, we take the symmetry-forbidden $\mathrm{H}_{2}+\mathrm{D}_{2}$ cycloaddition reaction via a $D_{4 h}$-symmetrical transition state leading to $2 \mathrm{HD}$ molecules (for reasons of simplicity we discuss the degenerate reaction $\mathrm{H}_{2}+\mathrm{H}_{2}$ ). This is a well-known example for the failure of standard Kohn-Sham DFT as was demonstrated by Baerends and co-workers. [5b] The reaction barrier can only be described by mixing the CSF of the ground state $A_{1 g}\left[\left(a_{1 g}\right)^{2}\left(b_{2 u}\right)^{2}\right]$ with the CSF of the doubly excited state $A_{1 g}\left[\left(a_{1 g}\right)^{2}\left(b_{3 u}\right)^{2}\right]$.

In Figures 3 and 4, the electronic structure of the reaction complex $\{\mathrm{H} 1-\mathrm{H} 2 \cdots \mathrm{H} 3-\mathrm{H} 4\}$ is described at $\mathrm{HF}$ and LDA theory with Dunning's cc-pVTZ basis set using difference densities (Figure 3) and one- or two-dimensional representations of the exchange hole (Figure 4) for a situation close to the transition state $(\mathrm{R}(\mathrm{H} 1-\mathrm{H} 2)=1.212$; $\mathrm{R}(\mathrm{H} 2-\mathrm{H} 4)=1.244 \AA)$. In the transition state, the bonds $\mathrm{H} 1-\mathrm{H} 2$ and $\mathrm{H} 3-\mathrm{H} 4$ are cleaved, while new bonds are formed between $\mathrm{H} 1$ and $\mathrm{H} 3$ as well as $\mathrm{H} 2$ and $\mathrm{H} 4$.

The difference density distribution $\Delta \rho(\mathrm{BS}-\mathrm{UHF}, \mathrm{RHF})=\rho(\mathrm{BS}-\mathrm{UHF})-\rho(\mathrm{RHF})$ (Figure $3 \mathrm{a})$ indicates the changes in the density due to the multi-reference effects included via the two-configurational approach of BS-UHF. [9] Density is shifted from the bonding regions $\mathrm{H} 1-\mathrm{H} 2$ and $\mathrm{H} 3-\mathrm{H} 4$ to the nonbonded regions $\mathrm{H} 1-\mathrm{H} 3$ and H2-H4 to support the formation of the new bonds. We can quantify this effect by calculating the fractional occupation numbers (FON) of the $b_{2 u}$-symmetrical HOMO and the $b_{3 u}$-symmetrical LUMO obtained in the BS-UHF calculations: 0.931 and 0.068 , respectively.

As found in the case of the dissociating $\mathrm{H}_{2}$ molecule the exchange functional mimics multi-reference effects. Accordingly, the same pattern of density changes is obtained for the difference density distribution $\triangle \rho(R L D A, R H F)=$ $\rho(R L D A)-\rho(R H F)$ (Figure $3 \mathrm{~b}$ ) as in the case of the BSUHF/RHF comparison (Figure 3a). Hence, RLDA plays the role of the BS-UHF, only that the long-range correlation effects mimicked by the exchange functional are clearly stronger (Figures $3 \mathrm{a}$ and $3 \mathrm{~b}$ ) than those incorporated via the two-configurational form of the wave-function. Although BS-U theory does not use a two-configurational wave function directly it is correct to say that the multireference effects implicitly introduced via the self-interaction error of the exchange functional are stronger than the multi-reference effects explicitly included via the form of the wave function.

Figure $3 \mathrm{c}$ shows the difference density distribution $\Delta \rho(\mathrm{BS}-\mathrm{ULDA}, \mathrm{RLDA})=\rho(\mathrm{BS}-\mathrm{ULDA})-\rho(\mathrm{RLDA})$, which gives a reversed pattern of density changes. Long-range electron correlation mimicked by the BS-ULDA approach is weaker than that covered by the RLDA approach thus increasing the density in bond regions $\mathrm{H} 1-\mathrm{H} 2$ and $\mathrm{H} 3-\mathrm{H} 4$ but decreasing it in the nonbonded regions $\mathrm{H} 1-\mathrm{H} 3$ and $\mathrm{H} 2-\mathrm{H} 4$ relative to the RLDA description. This is contrary to the expectation that account of multi-reference effects by both the exchange functional and the two-configurational form of the wave function should be stronger than those accounted by one of these effects alone. This seeming contradiction is resolved when considering that the BS-UDFT solution mimics via its exchange functional less long-range correlation than RDFT does (see above). In addition, the long-range effects simulated by the exchange functional suppress the long-range effects that should be explicitly included via the wave function. Accordingly, the calculated FON values for HOMO and LUMO are 1.0913 and 0.9087 , respectively, indicating a smaller admixture of the LUMO (or the doubly excited state) than obtained at the BS-UHF level of theory (FON: 1.0685 and 0.9315 ).

The explicit inclusion of long-range correlation effects is largest at the HF level, second largest at the SIC/LDA level, and smallest at the LDA level of theory as one can verify by comparing energy differences RHF-UHF, RSIC/LDA-BS-USIC/LDA, and RLDA - BSULDA for the same R(H-H) value (> bifurcation distance) in Figure 1. 
(a)

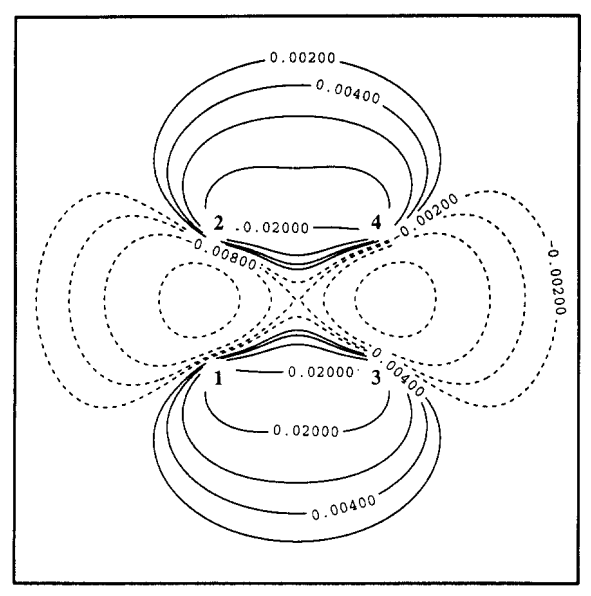

(c)

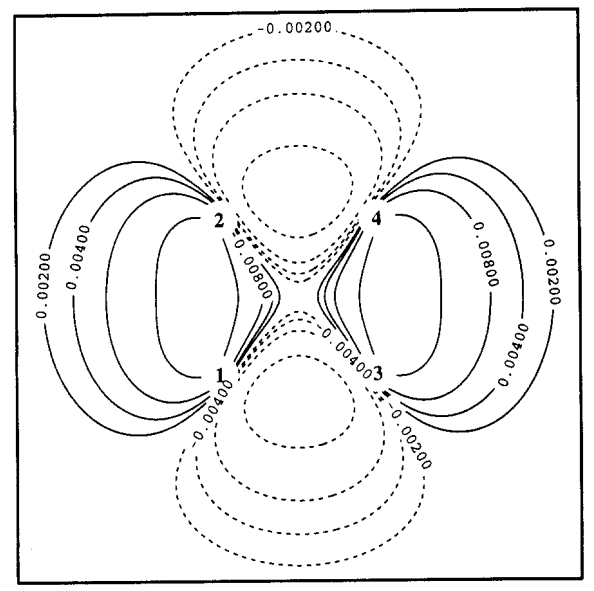

(b)

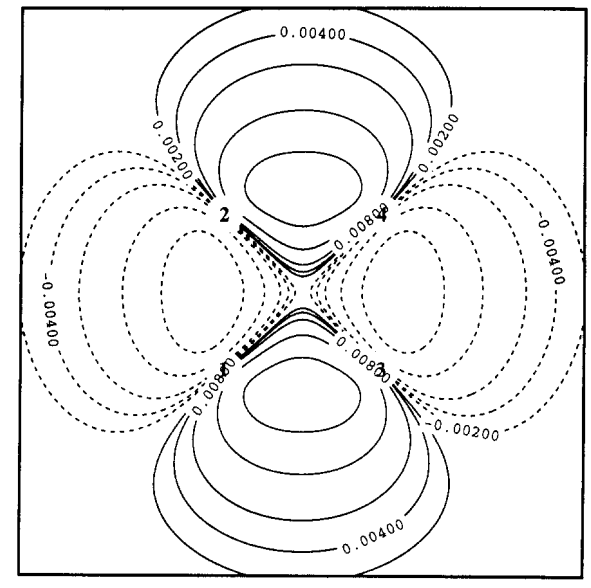

(d)

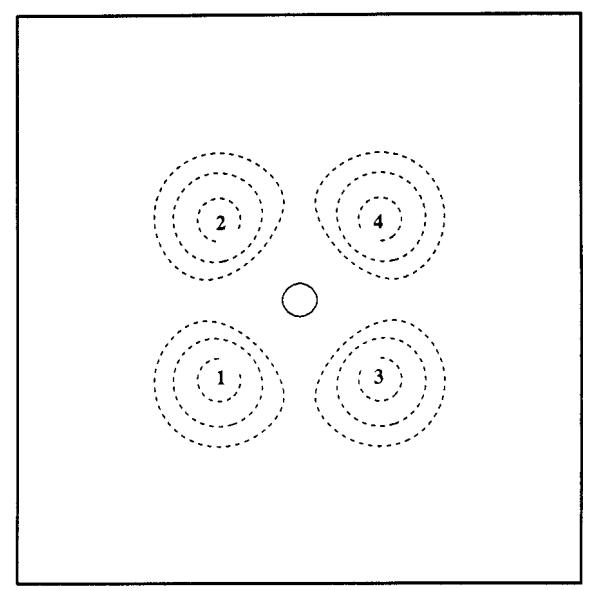

Figure 3: Contour line diagram of the difference electron density distribution $\Delta \rho(\mathbf{r})=\rho(\operatorname{method} \mathrm{I})-\rho(\operatorname{method}$ II) of the reaction complex $\mathrm{H}_{2}+\mathrm{H}_{2}$ calculated with the cc-pVTZ basis at a geometry close to the transition state $(\mathrm{R}(\mathrm{H} 1-\mathrm{H} 2)=1.212 ; \mathrm{R}(\mathrm{H} 2-\mathrm{H} 4)=1.244 \AA)$. Solid (dashed) contour lines are in regions of positive (negative) difference densities. Reference plane is the plane containing the four nuclei. The positions of the atoms are indicated by numbers 1 to 4 . The contour line levels have to be multiplied by the scaling factor 0.01 and are given in e bohr $^{-3}$. a) Method I: BS-UHF; method II: RHF. b) Method I: RLDA; method II: RHF. c) Method I: BS-ULDA; method II: RLDA. d) Method I: BS-ULDA; method II: BS-UHF. The LDA calculations were carried out with the Slater exchange-only functional. 
(a)

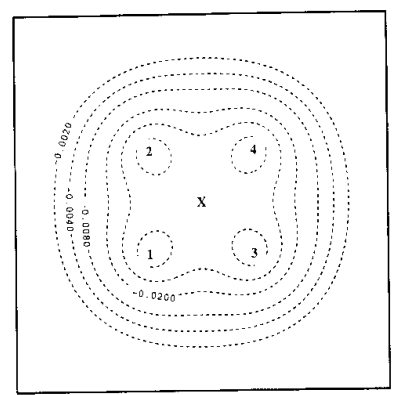

(c)

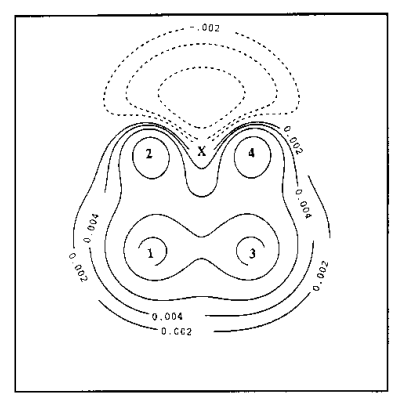

(b)

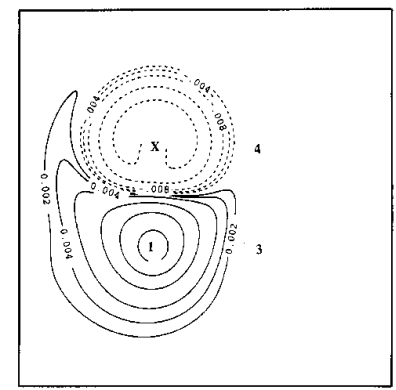

(d)

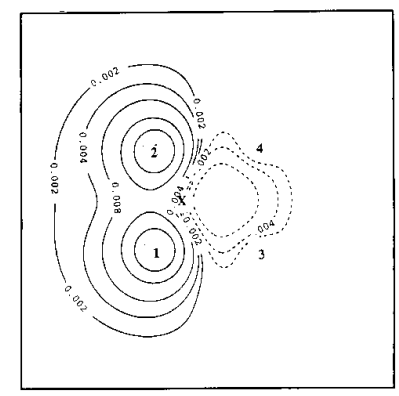

(e)

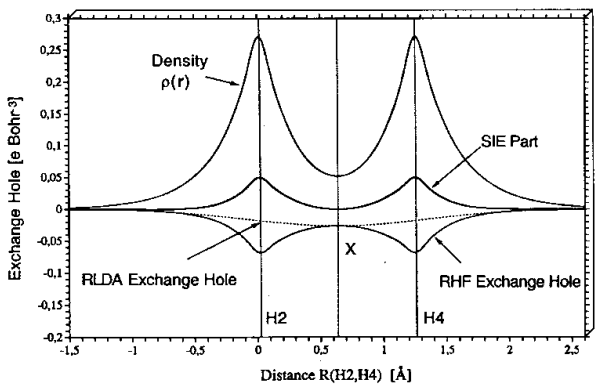

Figure 4: Contour line diagrams of the exchange hole of the reaction complex $\mathrm{H}_{2}+\mathrm{H}_{2}$ calculated with the cc-pVTZ basis at a geometry close to the transition state $(\mathrm{R}(\mathrm{H} 1-\mathrm{H} 2)=1.212 ; \mathrm{R}(\mathrm{H} 2-\mathrm{H} 4)=1.244 \AA)$. The contour line levels have to be multiplied by the scaling factor 0.01 and are given in e bohr ${ }^{-3}$. a) RHF exchange hole. The reference electron $(\mathrm{X})$ is at the center of the reaction complex. $\mathbf{b}-\mathbf{d}$ ) The part of the Slater exchange hole due to the self-interaction error. The reference electron X is at nucleus H2 (b), between H2 and H4 (c) or close to the bond H1-H2 (d). e) One-dimensional representation of the exchange hole calculated along the internuclear axis H2,H4. The reference electron $\mathrm{X}$ has the same position as in $\mathbf{c}$. 
Hence, the difference density distribution $\Delta \rho$ (BSULDA,BSUHF $)=\rho($ BS-ULDA $)-\rho($ BSUHF) $($ Figure $3 \mathrm{~d})$ indicates depletion of density in the nuclear regions.

In Figure 4a, the RHF exchange hole is shown in a two-dimensional (contour-line) representation again for the geometry close to the transition state of the $\mathrm{H}_{2}+\mathrm{H}_{2}$ cycloaddition reaction. The reference electron (denoted by $\mathrm{X}$ ) is at the center of the reaction complex. The exchange hole is delocalized possessing deep pockets at the positions of the four nuclei. For a vertical movement (toward the center of the H1,H3-distance or toward the center of the $\mathrm{H} 2, \mathrm{H} 4$ distance) of the reference electron, the RHF exchange hole is static while for a horizontal movement (toward the center of the H1-H2 bond or toward the center of the H3-H4 bond) of the reference electron the RHF exchange hole of the dissociating $\mathrm{H} 1-\mathrm{H} 2$ or the dissociating H3-H4 molecule is approached (see Figure 2a).

The RLDA exchange hole follows the reference electron and is always localized. It becomes very shallow and large in diameter in the nonbonded regions between $\mathrm{H} 1(\mathrm{H} 2)$ and $\mathrm{H} 3(\mathrm{H} 4)$ because of the smaller density in these regions (the outer dashed lines in Figure 4c provide an impression of the radius of the RLDA exchange hole). Hence, the self-interaction error part of the RLDA exchange hole adopts the forms shown in Figures 4b, 4c, and $4 \mathrm{~d}$. When the reference electron is at nucleus H2 (Figure 4b), there is a large probability of finding the second electron close to nucleus H1, i.e. strong left-right correlation effects are mimicked in bond H1-H2.

When the reference electron adopts a central position in the non-bonded region between H2 and H4 (Figure 4c), the probability of finding the second electron is large at $\mathrm{H} 2$ and $\mathrm{H} 4$, even larger at $\mathrm{H} 1$ and $\mathrm{H} 3$, but also in the nonbonded region between them. Again, the structure of the self-interaction part of the exchange hole suggests strong long-range left-right correlation effects in line with those found for the dissociating $\mathrm{H}_{2}$ molecule. Any movement of the reference electron from the central position adopted in Figure 4a to the left or right (Figure 4d) changes the situation to that of the corresponding $\mathrm{H}_{2}$ molecule approached, i.e. the part of the exchange hole due to the self-interaction error becomes identical to that shown in Figure 2a. Hence, RLDA with the SVWN5 exchangecorrelation functional can provide an improved, although not correct description of the $\mathrm{H}_{2}+\mathrm{H}_{2}$ cycloaddition reaction because multi-reference effects are simulated by the Slater exchange functional.

The observations made for the LDA exchange functional are equally valid for GGA exchange functionals. However, long-range correlation effects mimicked by the latter functionals are smaller because the self-interaction error is smaller in this case. [14] Also, the GGA exchange hole does not follow the reference electron in the region of low density so that the self-interaction part of the exchange hole differs significantly from the structure shown in Figure 4c. Nevertheless, the self-interaction error determines the stability of an RDFT solution and influences the extent of multi-reference effects incorporated explicitly via the form of the wave function into DFT. Therefore, we have to consider next whether implicit and explicit inclusion of multi-reference effects can lead to a conflicting description of long-range electron correlation, for example by a double-counting of correlation effects.

\section{Explicit Coverage of Multi-reference Effects: Ensemble DFT}

Since the advent of DFT [1] it was tacitly assumed that any ground state density can be represented by a single Slater determinant constructed from the N lowest eigenfunctions of a certain non-interacting Hamiltonian (10)

$$
\hat{H}=\sum_{i}-\frac{1}{2} \nabla^{2}+V_{s}(\mathbf{r})
$$


where the first term on the right side represents the kinetic energy part and the second the external potential. This assumption is embodied into the Kohn-Sham formulation of DFT [1b] where an auxiliary system of noninteracting electrons, which possesses the ground state density identical to the density of a target system, plays a crucial role. The existence of the corresponding potential, $V_{s}$ in eq. (10), has been demonstrated utilizing the adiabatic connection between the real system of interacting electrons and a fictitious system with the interelectronic interaction switched off $[41,42]$. Such an adiabatic route does exist only if the corresponding density at the intermediate or zero electron-electron coupling strength is the ground state density [27].

However, it may well be that certain atomic or molecular electron density situations cannot be described by the ground state of a certain non-interacting Hamiltonian as was discussed early in the literature [27,28]. To circumvent the problem of $V$-representability it was suggested $[27,28]$ to use the ensemble density (11)

$$
\rho_{e}(\mathbf{r})=\sum_{i} w_{i} \rho_{i}(\mathbf{r})
$$

$\left(w_{i}\right.$ : weighting factor for state $i$ with density $\left.\rho_{i}\right)$ rather than the density of a single Slater determinant. Indeed, theoretical arguments were given that the ensemble representation of the density is the only one that guarantees the existence of the corresponding potential $V_{s}$ in eq. (10). In terms of the one-electron orbitals, the ensemble representation translates to the fractional occupation numbers of the orbitals $\phi_{k}(\mathbf{r}), i$. $e$.

$$
\rho_{e}(\mathbf{r})=\sum_{k} n_{k}\left|\phi_{k}(\mathbf{r})\right|^{2}, \quad 0 \leq n_{k} \leq 2 .
$$

However, despite the formal exactness of the ensemble representation of the density, until recently it was considered to be of purely academic interest. Recently, the long-standing assumption that any non-degenerate ground state density can be described by a single ground state determinant was rejected by the results of accurate numeric Kohn-Sham simulations of the exact densities by Baerends and co-workers [5]. These authors demonstrated that for the ${ }^{1} \Sigma_{g}^{+}$ground state of the $C_{2}$ molecule and for the ${ }^{1} A_{g}$ ground state of the $H_{2}+H_{2}$ system it is impossible to fit the KS density to the exact one unless fractional occupation numbers are invoked for certain KS orbitals. Taken together with the theoretical arguments, these results showed unambiguously that the ensemble representation has practical relevance and is the only rigorous representation for the density of a system with strong multi-reference character.

Ensemble DFT has a number of advantages. First of all, it enables one to calculate systems with strong multireference effects on the same footing as systems without these effects. In the latter case, the ensemble representation merely collapses to the single determinant as in the conventional Kohn-Sham method. For systems with strong nondynamic electron correlation, the ground state density is represented by more than one Kohn-Sham determinant within the ensemble DFT approach. In principle, the selection of the determinants for the ensemble is performed automatically when minimizing the ensemble DFT energy functional with respect to the ensemble DFT density. Only the Kohn-Sham orbitals, which are degenerate at the Fermi level will be fractionally populated and the FONs will be obtained. However, this implies the use of the exact Kohn-Sham energy functional constructed for the ensemble densities. Such a functional should conform with the sum rules for the exchange-correlation hole and the on-top pair densities valid for the ensemble densities. The conventional approximate density functionals are based on the single determinant density representation and can not directly be used in the ensemble KS method.

Formally, the ensemble DFT approach can be formulated for a treatment of excited states as well. However, special density functionals must also be used in this case. Thus, it is the lack of the density functionals conforming 
with the ensemble representation of the density that hinders the practical implementation of ensemble DFT. It must be stressed that, contrary to general belief, the knowledge of the exact density functionals for the exchangecorrelation energy would not guarantee an exact description of systems with strong multi-reference effects within standard single determinant DFT.

The REKS method [29,30] fills this gap in computational DFT. The method combines some ideas from WFT for constructing the energy functional in case of an ensemble while keeping the exact ensemble representation for the density. The density in REKS is represented as an average over densities of several configurations described by single determinants. In case of the $\operatorname{REKS}(2,2)$ method, there are two configurations: one with doubly occupied orbital $\phi_{r}$ and another with doubly occupied orbital $\phi_{s}$, where $\phi_{r}$ and $\phi_{s}$ can be the HOMO and the LUMO from the conventional single determinant Kohn-Sham calculation. The inactive core orbitals are occupied with 2 electrons each, such that the ground state density is given by eq. (13).

$$
\rho^{R E K S}(\mathbf{r})=\sum_{j}^{\text {core }} 2\left|\phi_{j}(\mathbf{r})\right|^{2}+n_{r}\left|\phi_{r}(\mathbf{r})\right|^{2}+n_{s}\left|\phi_{s}(\mathbf{r})\right|^{2}
$$

The total ground state energy for a state with two fractionally occupied orbitals is represented as a weighted sum of Kohn-Sham energies, $E^{K S}\left(\ldots \phi_{r}^{2} \ldots\right)$ and $E^{K S}\left(\ldots \phi_{s}^{2} \ldots\right)$ of the two configurations described above and a coupling term $D\left(\phi_{r}, \phi_{s}\right)$ which is expressed as a linear combination of KS energies of the singly excited configurations generated within the same $(2,2)$ active space.

$$
E^{R E K S(2,2)}=\frac{n_{r}}{2} E^{K S}\left(\ldots \phi_{r}^{2} \ldots\right)+\frac{n_{s}}{2} E^{K S}\left(\ldots \phi_{s}^{2} \ldots\right)+\left(n_{r} n_{s}\right)^{3 / 4} D\left(\phi_{r}, \phi_{s}\right)
$$

The dependence of the weighting factors in eq. (5) on the fractional occupation numbers of the active orbitals was derived from model considerations, [29] which are similar to those used by von Barth [8] when developing DFT for the multiplet states. Special care was taken to guarantee that at the two limiting situations, namely the 'normal' system with $n_{r} \approx 2$ and $n_{s} \approx 0$ and open-shell singlet system with $n_{r}=n_{s}=1$, the results of the conventional spin-restricted Kohn-Sham calculations are reproduced by REKS. [29] The one-electron orbitals and the FON values are obtained in REKS variationally from minimization of the REKS total energy (14). [29]

The model nature of the REKS energy expression makes it possible to use standard density functionals in a REKS calculation. Thus, REKS has an advantage of not being bound to a certain specific density functional. However, the same model nature of REKS makes further extension of the 'active' space quite difficult. While the REKS concept can be relatively easy extended to include the case of three electrons in three orbitals, i.e. $\operatorname{REKS}(3,3)$, [29] the treatment of more complicated situations such as double bond dissociation, which includes four electrons in four orbitals, is not as easy. Furthermore, there is no automatic way of extending the REKS 'active' space, like in CASSCF theory, and one needs to derive the energy expressions separately for every case of interest.

The fractional occupation numbers of the orbitals in REKS are analogous to the natural orbital occupation numbers (NOONs) in conventional multi-reference theory. Thus, the concepts developed in WFT for the analysis of the multi-reference wavefunctions can be applied to the analysis of the REKS density and energy. The oneelectron properties in REKS can be straightforwardly obtained from the density given by Eq. (13). The energy gradient with respect to atomic coordinates is also obtained easily from differentiation of Eq. (14). Thus, it is the feasibility of the REKS method that makes it an attractive alternative to the conventional multi-reference 


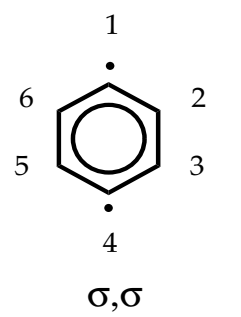

$1, \mathrm{D}_{2 \mathrm{~h}}$

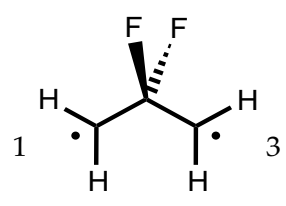

$\pi, \pi$

$4, \mathrm{C}_{2 \mathrm{v}}$

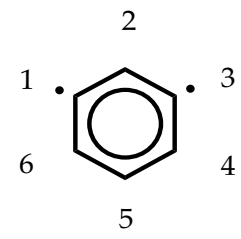

$\sigma, \sigma$

$2, \mathrm{C}_{2 \mathrm{v}}$

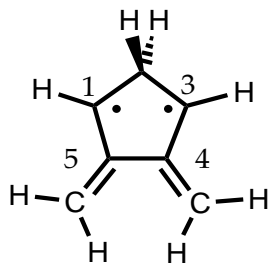

$\pi, \pi$

$5, \mathrm{C}_{2 \mathrm{v}}$

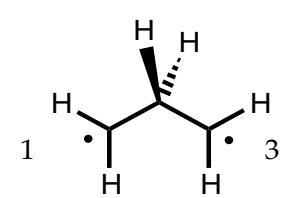

$\pi, \pi$

$3, \mathrm{C}_{2 \mathrm{v}}$

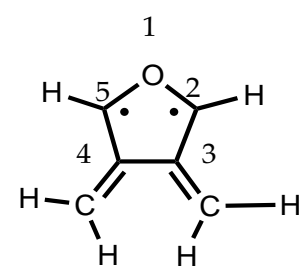

$\pi, \pi$

$6, \mathrm{C}_{2 \mathrm{v}}$

Scheme 1: Biradicals investigated in this work.

methods in WFT, especially in application to large molecular systems. Therefore, we will consider in the next section how REKS adjusts the explicit account of multi-reference effects to the implicit introduction of the latter via the exchange functional. For this purpose, we present for the first time REKS calculations based on the use of an hybrid functional, which were not possible with the previous version of the program. [29]

\section{Implicit and Explicit Coverage of multi-reference Effects: The Problem of Double Counting of Electron Correlation}

Singlet-triplet splittings $\Delta E_{S T}$ were calculated for a number of biradicals with distinct multi-reference character both with restricted Kohn-Sham DFT, BS-UDFT, and the ensemble DFT REKS(2,2) method. The biradicals investigated (see Scheme 1) include p-didehydrobenzene (p-benzyne, 1), m-didehydrobenzene (m-benzyne, 2), propane-1,3-diyl (trimethylene, 3), 2,2-difluoropropane-1,3-diyl (4), 4,5-dimethylene-cyclopentane-1,3-diyl (5), and 3,4-dimethylene-oxolane-2,5-diyl (6). The open-shell singlet states of these molecules represent type II systems, which can be described by mixing the ground state with the doubly-excited state formed by exciting two electrons from the HOMO to the LUMO, i.e. the biradical character obtained by this two-configurational description can be 
assessed from the FON values of HOMO and LUMO. The triplet states are high-spin systems without any multireference character (type 0 systems). They constitute appropriate reference systems, which are needed to assess the multi-reference effects of the singlet biradicals. Note that for some of these molecules, REKS/BLYP calculations based on the 6-31G(d) basis set were previously published. [29]

Pople's 6-31G(d) basis [43] and Dunning's cc-pVTZ basis [35] were used in connection with three different functionals, namely a) BLYP $[44,45]$ as an example for a GGA functional characterized by a relatively large self-interaction error in the exchange part, b) B3LYP [46] as an example for a hybrid functional with a considerably reduced self-interaction error due to the admixture of $20 \%$ HF exchange, and c) BH\&HLYP [47] as an example for a hybrid functional with a relatively small self-interaction error due to an admixture of $50 \% \mathrm{HF}$ exchange. In addition, some reference calculations were carried out with the LDA functional SVWN5 [36,37]. In cases a), b), c) the correlation functional LYP is without a self-interaction error which for other correlation functionals such as VWN, [37] VWN5 [37] or PW91 [48] lead to a negative correlation energy in the case of a single electron. The LYP correlation functional [45] was constructed from the Colle-Salvetti functional [49] that as a WFT-based functional is free of any residual self-interaction terms. - All calculations were carried out with the program packages COLOGNE2001 [50] and Gaussian98. [51]

Table 1. Description of p-benzyne (1) at different levels of DFT. ${ }^{a}$

\begin{tabular}{ccccccccc}
\hline \multicolumn{1}{c}{} & \multicolumn{1}{c}{ RDFT } & \multicolumn{3}{c}{ BS-UDFT } & \multicolumn{3}{c}{ REKS(2,2) } \\
& $\Delta E_{S T}$ & $\Delta$ & $\Delta E_{S T}$ & $\Delta$ & $\%$ Birad & $\Delta E_{S T}$ & $\Delta$ & $\%$ Birad \\
& & & & & & & & \\
\hline \multirow{3}{*}{ SVWN5 } & -3.7 & 0.087 & -5.7 & 0.047 & 39 & -6.4 & 0.064 & 19 \\
& $(-5.8)$ & $(0.091)$ & $(-6.6)$ & $(0.059)$ & $(34)$ & $(-7.2)$ & 0.070 & $(16)$ \\
BLYP & 1.3 & 0.097 & -4.1 & 0.037 & 51 & -5.0 & 0.056 & 32 \\
& $(-0.1)$ & $(0.105)$ & $(-4.5)$ & $(0.041)$ & $(50)$ & $(-5.7)$ & $(0.063)$ & $(28)$ \\
B3LYP & 14.9 & 0.106 & -2.4 & 0.019 & 74 & -2.1 & 0.031 & 59 \\
& $(13.1)$ & $(0.109)$ & $(-2.6)$ & $(0.022)$ & $(72)$ & $(-2.5)$ & $(0.033)$ & $(57)$ \\
BH\&HLYP & 36.7 & 0.117 & -1.8 & 0.010 & 84 & -0.7 & 0.015 & 77 \\
& $(34.3)$ & $(0.120)$ & $(-1.7)$ & $(0.010)$ & $(84)$ & $(-0.8)$ & $(0.018)$ & $(76)$ \\
& & & & & & & & \\
\hline
\end{tabular}

${ }^{a}$ Calculations with the 6-31G(d) basis; numbers in parentheses with Dunning's cc-pVTZ basis. Energy differences $\Delta E_{S T}$ between singlet and triplet state in $\mathrm{kcal} / \mathrm{mol}$ (a negative value indicates that the singlet state is more stable), $\Delta$ is the difference $[R(C 2-C 3)-R(C 2-C 1)]^{S}-[R(C 2-C 3)-R(C 2-C 1)]^{T}$ given in $\AA$, \% Birad gives the calculated biradical character in percent as $100 \times \mathrm{FON}(\mathrm{LUMO})$. 
In Tables 1 and 2, calculated singlet-triplet splittings are listed for biradicals $\mathbf{1}$ - 6. Also given are the FON values of HOMO and LUMO (Table 2), which reflect the biradical character and, by this, the amount of long-range correlation effects introduced by REKS(2,2). Calculated REKS geometries are given in Figure 5 .

The exchange functionals of DFT cover long-range correlation effects, which keep the two unpaired electrons apart and thereby reduce the need for spin-coupling via through-bond interactions. Accordingly, the parameter $\Delta$ increases for restricted theory in the series (Table 1)

$$
S V W N 5<B L Y P<B 3 L Y P<B H \& H L Y P<H F
$$

since the long-range effects mimicked by the exchange functional decrease in this order. Enforced through-bond coupling implies strained geometries that convert 1 into two allyl units hold together by two long C2-C3/C5-C6 bonds that disturb $\pi$-delocalization and destabilize the molecule. Consequently, the singlet state is higher in energy than the triplet state, which benefits from the exchange interactions of the two unpaired electrons with aligned spin (thus reducing Coulomb repulsion). This is reflected by the calculated singlet-triplet splittings of -3.7 (SVWN5), 1.3 (BLYP), 14.9 (B3LYP), and $36.7 \mathrm{kcal} / \mathrm{mol}$ (BH\&HLYP; all calculations with the 6-31G(d) basis; for cc-pVTZ results, see Table 1), respectively where the triplet state was calculated at the UDFT level of theory.

Table 2. Singlet-triplet energy splitting $\Delta E_{S T}$ and fractional occupation numbers (FON) of orbitals as calculated with the $\operatorname{REKS}(2,2)$ method using different functionals and basis sets. ${ }^{a}$

\begin{tabular}{|c|c|c|c|c|c|c|c|c|c|c|c|}
\hline Molecule & $\Delta E_{S T}$ & $\begin{array}{l}\text { BLYP } \\
F O N \\
\text { MO1 }\end{array}$ & $\begin{array}{l}F O N \\
\mathrm{MO} 2 \\
\end{array}$ & $\Delta E_{S T}$ & $\begin{array}{c}\text { B3LYP } \\
F O N \\
\text { MO1 }\end{array}$ & $\begin{array}{l}\text { FON } \\
\mathrm{MO} 2 \\
\end{array}$ & $\Delta E_{S T}$ & $\begin{array}{c}\text { BH\&H- } \\
\text { LYP } \\
F O N \\
\text { MO1 } \\
\end{array}$ & $\begin{array}{r}F O N \\
\mathrm{MO} 2 \\
\end{array}$ & $\begin{array}{c}\text { Ref. } \\
\text { Value } \\
\Delta E_{S T}\end{array}$ & Ref. \\
\hline $\begin{array}{c}1 \\
p-C_{6} H_{4}\end{array}$ & $\begin{array}{l}-5.0 \\
(-5.7)\end{array}$ & $\begin{array}{c}1.68 \\
(1.72)\end{array}$ & $\begin{array}{c}0.32 \\
(0.28)\end{array}$ & $\begin{array}{l}-2.1 \\
(-2.5)\end{array}$ & $\begin{array}{c}1.41 \\
(1.43)\end{array}$ & $\begin{array}{c}0.59 \\
(0.57)\end{array}$ & $\begin{array}{l}-0.7 \\
(-0.8)\end{array}$ & $\begin{array}{c}1.23 \\
(1.24)\end{array}$ & $\begin{array}{c}0.77 \\
(0.76)\end{array}$ & 3.5 & 60 \\
\hline 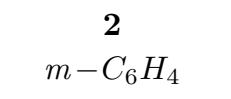 & $\begin{array}{l}-21.9 \\
(-22.0)\end{array}$ & $\begin{array}{c}1.96 \\
(1.97)\end{array}$ & $\begin{array}{c}0.04 \\
(0.03)\end{array}$ & $\begin{array}{c}-16.6 \\
(-17.0)\end{array}$ & $\begin{array}{c}1.88 \\
(1.90)\end{array}$ & $\begin{array}{c}0.12 \\
(0.10)\end{array}$ & $\begin{array}{c}-11.4 \\
(-11.7)\end{array}$ & $\begin{array}{c}1.62 \\
(1.65)\end{array}$ & $\begin{array}{c}0.38 \\
(0.35)\end{array}$ & 21.1 & 60 \\
\hline $\begin{array}{c}\mathbf{3} \\
\mathrm{C}_{3} \mathrm{H}_{6}\end{array}$ & $\begin{array}{c}0.7 \\
(0.1)\end{array}$ & $\begin{array}{c}1.36 \\
(1.47)\end{array}$ & $\begin{array}{c}0.64 \\
(0.53)\end{array}$ & $\begin{array}{c}1.0 \\
(0.8)\end{array}$ & $\begin{array}{c}1.21 \\
(1.27)\end{array}$ & $\begin{array}{c}0.79 \\
(0.73)\end{array}$ & $\begin{array}{l}1.0 \\
(1.0)\end{array}$ & $\begin{array}{c}1.12 \\
(1.15)\end{array}$ & $\begin{array}{c}0.88 \\
(0.85)\end{array}$ & $0.7^{b}$ & 62 \\
\hline $\begin{array}{c}4 \\
C_{3} H_{4} F_{2}\end{array}$ & $\begin{array}{l}-5.5 \\
(-7.0)\end{array}$ & $\begin{array}{c}1.66 \\
(1.75)\end{array}$ & $\begin{array}{c}0.34 \\
(0.25)\end{array}$ & $\begin{array}{l}-3.7 \\
(-4.6)\end{array}$ & $\begin{array}{c}1.45 \\
(1.51)\end{array}$ & $\begin{array}{c}0.55 \\
(0.49)\end{array}$ & $\begin{array}{l}-2.7 \\
(-3.2)\end{array}$ & $\begin{array}{c}1.31 \\
(1.35)\end{array}$ & $\begin{array}{c}0.69 \\
(0.65)\end{array}$ & $-4.8^{b}$ & 62 \\
\hline $\begin{array}{c}\mathbf{5} \\
\mathrm{C}_{5} \mathrm{H}_{4}\left(\mathrm{CH}_{2}\right)_{2}\end{array}$ & $\begin{array}{c}0.5 \\
(0.6)\end{array}$ & $\begin{array}{c}1.26 \\
(1.28)\end{array}$ & $\begin{array}{c}0.74 \\
(0.72)\end{array}$ & $\begin{array}{c}0.7 \\
(0.7)\end{array}$ & $\begin{array}{c}1.15 \\
(1.17)\end{array}$ & $\begin{array}{c}0.85 \\
(0.83)\end{array}$ & $\begin{array}{c}0.6 \\
(0.6)\end{array}$ & $\begin{array}{c}1.10 \\
(1.12)\end{array}$ & $\begin{array}{c}0.90 \\
(0.88)\end{array}$ & $>0$ & 64 \\
\hline $\begin{array}{c}\mathbf{6} \\
O C_{4} H_{2}\left(C_{2}\right)_{2}\end{array}$ & $\begin{array}{l}-4.7 \\
(-4.7)\end{array}$ & $\begin{array}{c}1.86 \\
(1.89)\end{array}$ & $\begin{array}{c}0.14 \\
(0.11)\end{array}$ & $\begin{array}{l}-2.8 \\
(-2.5)\end{array}$ & $\begin{array}{c}1.61 \\
(1.63)\end{array}$ & $\begin{array}{c}0.39 \\
(0.37)\end{array}$ & $\begin{array}{l}-1.6 \\
(-1.2)\end{array}$ & $\begin{array}{l}1.35 \\
(1.35)\end{array}$ & $\begin{array}{c}0.65 \\
(0.65)\end{array}$ & $<0$ & 66 \\
\hline
\end{tabular}

${ }^{a}$ Calculations with the $6-31 \mathrm{G}(\mathrm{d})$ basis set. In parentheses, results obtained with the cc-pVTZ basis set. Energy differences $\Delta E_{S T}$ between singlet and triplet state in $\mathrm{kcal} / \mathrm{mol}$ (a negative value indicates that the singlet state is more stable). MO1 corresponds to the HOMO, MO2 to the LUMO. - ${ }^{b}$ CASPT2 results. 
Restricted HF and SIC-DFT theory predict with regard to the two unpaired electrons a delocalized exchange hole stretching from C1 to C4 (2.7 $\AA$ ). Hence, the self interaction error of the localized RDFT hole is relatively large as are the long-range effects mimicked by it. REKS-DFT leads to a localized exchange hole similar to that shown in Figure $2 \mathrm{~b}$. The self-interaction error of the exchange functional simulates short-range correlation, which is added to that of the LYP correlation functional. Hence, a double-counting of dynamic electron correlation cannot be excluded. This, however, should not be the case for the long-range correlation effects, which may also be simulated by the exchange functional according to the analysis of the $\mathrm{H}_{2}$ case given above.

Implicit coverage of long-range effects via the exchange functional leads to a stabilization of the singlet state and a corresponding increase of $\Delta E_{S T}$. The energy difference between singlet ground state and the doubly excited state increases and, by this, the possibility of state mixing is reduced. State mixing in ensemble DFT leads to an occupation of the $a_{g}$-symmetrical LUMO (Scheme 2) which is C2-C3 and C5-C6 bonding thus reducing the influence of through-bond coupling. Thus, the parameter $\Delta$ reflects the amount of CSF-mixing and the corresponding reduction of through-bond coupling.

Clearly, these effects are moderate when using a BLYP functional (FON value for LUMO: 0.32), but increase when reducing the self-interaction error of the Becke exchange functional by admixing in HF exchange: Less longrange correlation effects are mimicked, the singlet energy increases (as documented by a more positive singlet-triplet splittings), the energy difference between singlet state and doubly-excited state decreases, and REKS leads to a larger admixture of the doubly-excited state as reflected by the FON values and the percent biradical character given by them: 59\% (B3LYP), 77\% (BH\&HLYP; 6-31G(d) basis, Table 1). Ensemble DFT leads to a stabilization of the singlet state relative to the triplet state by $6.2 \mathrm{kcal} / \mathrm{mol}$ (BLYP), $16.9 \mathrm{kcal} / \mathrm{mol}$ (B3LYP), and $37.3 \mathrm{kcal} / \mathrm{mol}$ (BH\&HLYP, Table 1). The stabilization energies caused by the explicit coverage of multi-reference effects increase in the same way as implicit effects are decreased due to a reduced self-interaction error of the exchange functional.

The trends observed for $\operatorname{REKS}(2,2)$ are parallel to those obtained with BS-UDFT as both approaches lead to two-configurational descriptions (Table 1). Singlet-triplet splittings close to the experimental $\Delta E_{S T}$ value of -3.5 $\mathrm{kcal} / \mathrm{mol}[60,53]$ are obtained with B3LYP using a larger basis set (BS/UDFT/cc-pVTZ: -2.6), REKS(2,2)/ccpVTZ: $-2.5 \mathrm{kcal} / \mathrm{mol}$, Table 1) while with the BLYP functional values of singlet-triplet splittings are the more exaggerated the larger the basis set used (-4.5 and $-5.7 \mathrm{kcal} / \mathrm{mol}$, Table 1$)$. Since a larger basis set should lead to the better results, we conclude that B3LYP provides the more reliable description. The biradical character predicted by REKS(2,2)/B3LYP (ca $60 \%$, Table 1) is comparable to that suggested on the basis of CCSD(T) calculations ( $65 \%$ [52b]) while the corresponding $\operatorname{REKS}(2,2) /$ BLYP value $(32 \%$, Table 1) is much smaller.

For 2, a through-space 1,3-bond can be established to avoid a biradical structure. [52,57-59] Hence, restricted theory with HF or hybrid functional falsely predicts bicyclo[3.1.0]hexatriene to be the only isomer of $\mathbf{2}$. [58,59] This is also true for those functionals that exaggerate the strength of a bond. Hence, LDA functionals, despite of the multi-reference effects mimicked by the Slater exchange functional, predict a bicyclic rather than biradical form thus indicating that the nature of the functional dominates.

GGA functionals such as BLYP predict weaker bonds and, by this, the long-range correlation effects mimicked by the Becke exchange functional can play a dominant role: They lead to a lengthening to the C1-C3 bond to ca. $2 \AA[58,59]$. The energy differences involved are relatively small: The stretching of the CC bond from 1.6 to $2 \AA$ leads to a $2.5 \mathrm{kcal} / \mathrm{mol}$ gain in energy at RBLYP, but a $1 \mathrm{kcal} / \mathrm{mol}$ loss in energy at RB3LYP, hence the difference in the two descriptions being about $3.5 \mathrm{kcal} / \mathrm{mol}$. [58] 

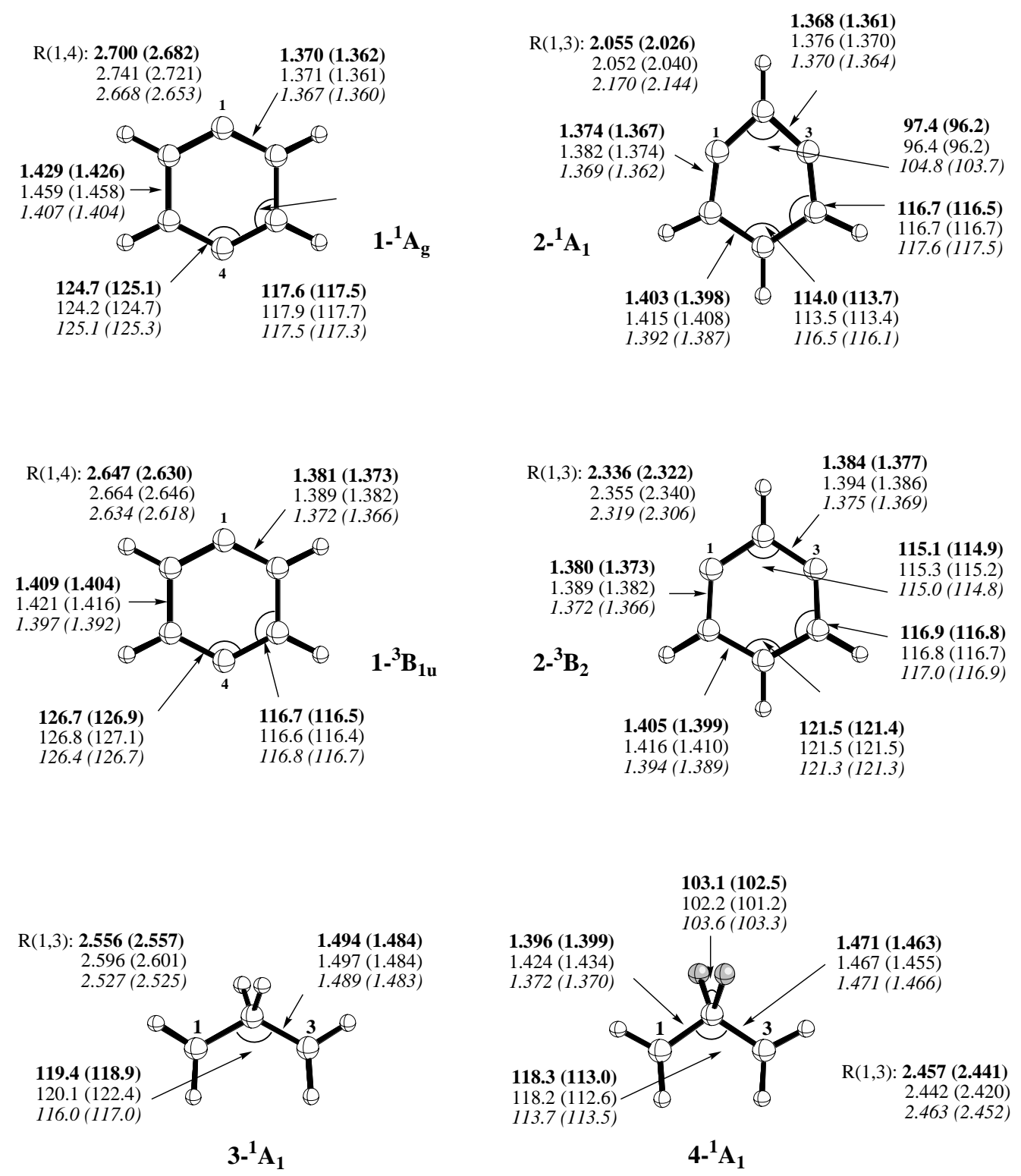

Figure 5: Parts a and b. For caption, see next page. 


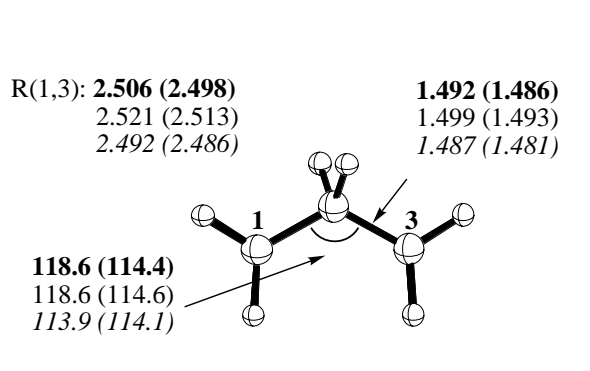

${ }^{3-}{ }^{3} \mathbf{B}_{2}$
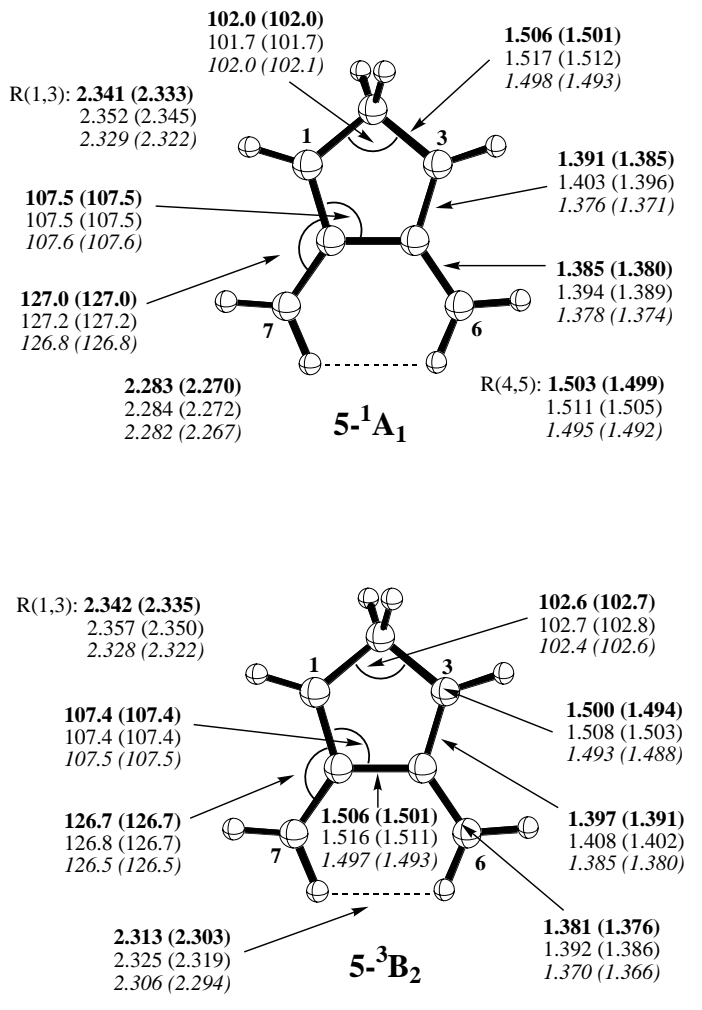

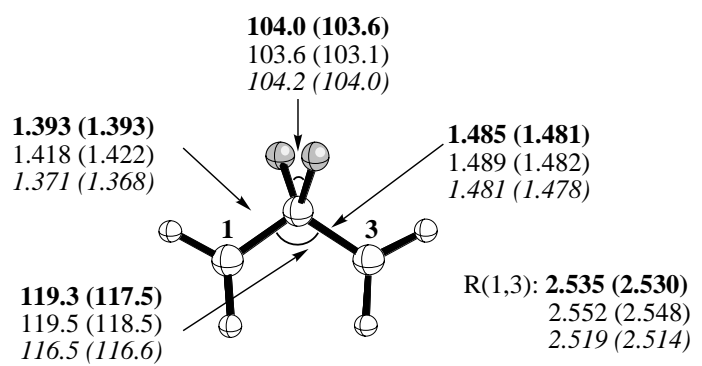

4- ${ }^{3} B_{2}$
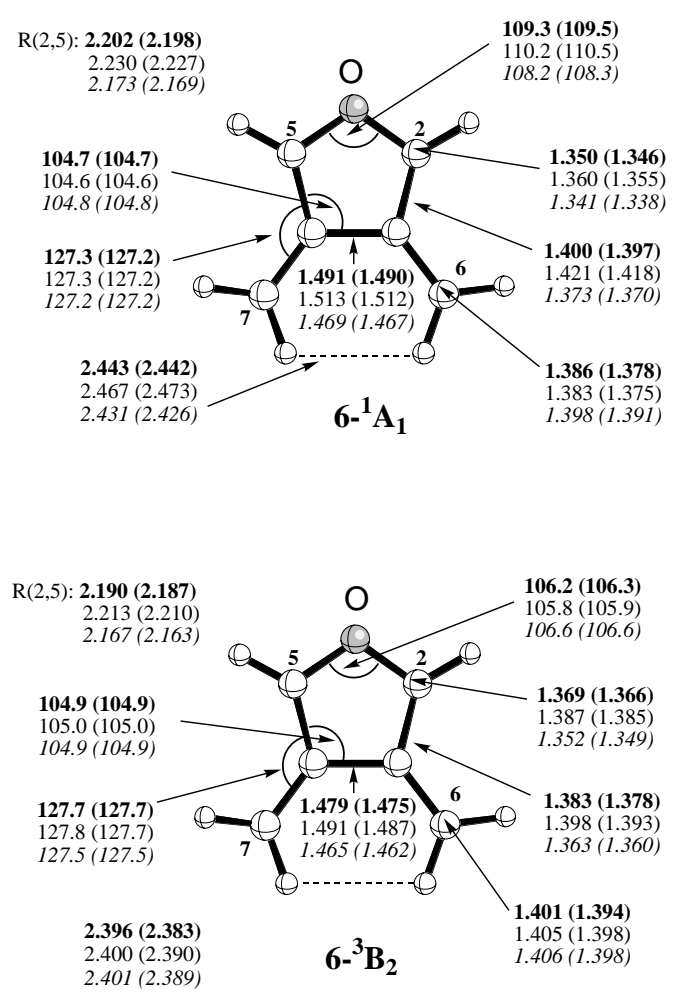

Figure 5: REKS $(2,2) / 6-31 \mathrm{G}(\mathrm{d})$ geometries of the lowest singlet and triplet state of biradicals 1 - 6. B3LYP: values in bold print, BLYP: normal print, BH\&HLYP: italics. Numbers in parentheses were obtained with the cc-pVTZ basis set. Distances in $\AA$, angles in degree. 

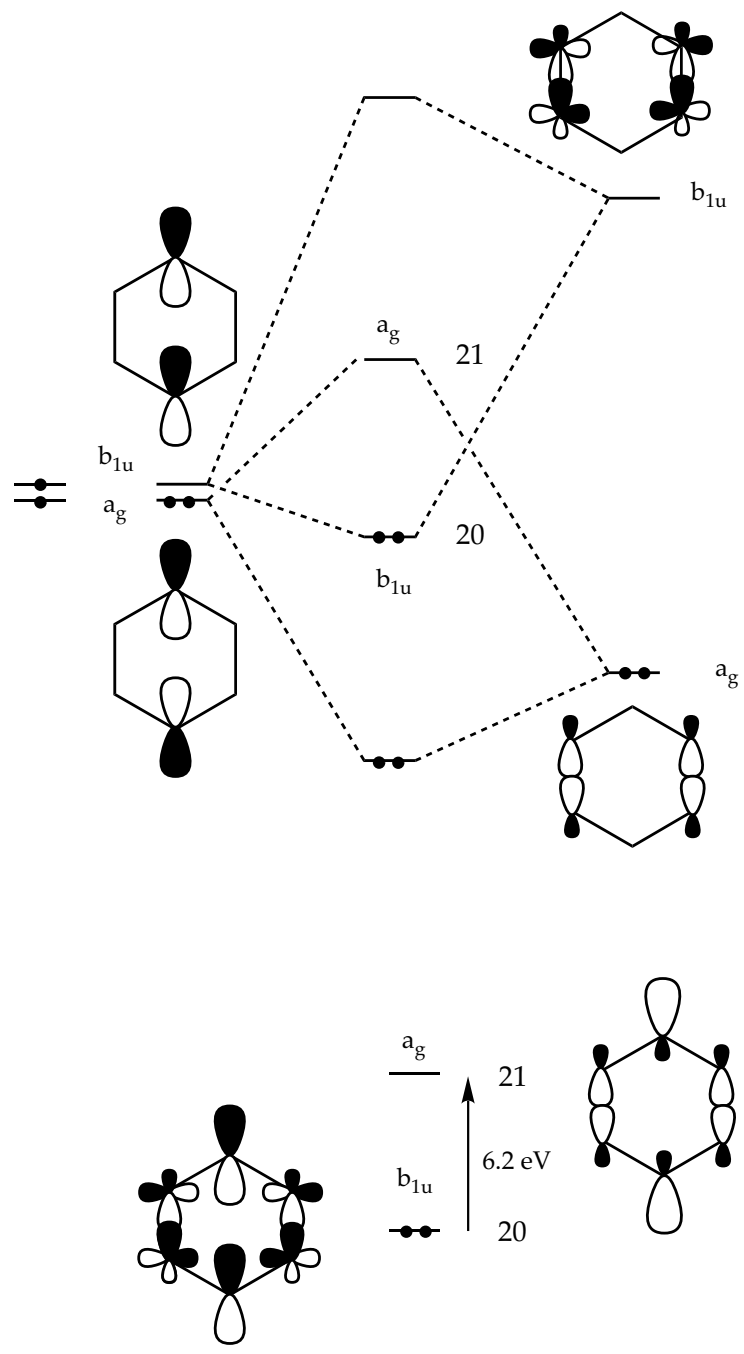

Scheme 2: Frontier orbitals of p-benzyne (1). a) Interactions of the orbitals occupied by the single electrons with the $\sigma\left(\mathrm{C} 2 \mathrm{C} 3\right.$;C5C6) and $\sigma^{\star}(\mathrm{C} 2 \mathrm{C} 3$; $5 \mathrm{C} 6)$ orbitals as needed for through-bond coupling between the single electrons. b) Frontier orbitals MO20 and MO21 of 1 resulting from the interactions shown in a) taken from a HF calculation. 
When multi-reference effects are included via $\operatorname{REKS}(2,2)$, they are smaller for $\mathbf{2}$ than for $\mathbf{1}$ by at least a factor of five (see FON values for B3LYP in Table 2), which reflects the fact that the biradical (multi-reference) character of $\mathbf{2}$ is considerably reduced compared to that of $\mathbf{1}$. Thus, the (absolute) corrections in the singlet-triplet splitting of 2 caused by REKS are smaller: a) BH\&HLYP: $-4 \mathrm{kcal} / \mathrm{mol}$ (from -7.4 to $-11.4 \mathrm{kcal} / \mathrm{mol}$, Table 2); b) B3LYP: $-2.3 \mathrm{kcal} / \mathrm{mol}$ (from -14.3 to $-16.6 \mathrm{kcal} / \mathrm{mol}$ ); c) BLYP: $-2.2 \mathrm{kcal} / \mathrm{mol}$ (from -19.7 to $-21.9 \mathrm{kcal} / \mathrm{mol}$ ).

Again, the changes in the singlet-triplet splitting caused by REKS increase with decreasing account of implicit multi-reference effects introduced by the exchange functional. However, when the latter correlation effects are not simulated by the exchange functional, the singlet-triplet splitting is largely underestimated. The REKS/BLYP results are just $1 \mathrm{kcal} / \mathrm{mol}$ more negative than the experimental value of $-21.1 \mathrm{kcal} / \mathrm{mol}$ for the singlet-triplet splitting [60] (the thermochemical corrections are just $0.4 \mathrm{kcal} / \mathrm{mol}$ ) while the corresponding REKS/B3LYP energy differences are $4 \mathrm{kcal} / \mathrm{mol}$ too positive. Therefore, one could draw the conclusion that the implicit account of longrange correlation improves the BLYP results relative to the B3LYP results. However, two additional considerations have to be made:

a) The error in the REKS/B3LYP singlet-triplet splitting of -17 kcal/mol (cc-pVTZ basis, Table 2) corresponds to the B3LYP destabilization energy of $3.5 \mathrm{kcal} / \mathrm{mol}$ caused by stretching the C1-C3 bond. Since the B3LYP functional [46] was optimized to reproduce the thermochemistry of suitable reference molecules and, by this, the strength of the bonds in these compounds, it makes little sense to draw the conclusion that the B3LYP functional, because of its local contributions, exaggerates the strength of the $\mathrm{C} 1 \mathrm{C} 3$ interactions and therefore has to be reoptimized.

b) A $(2,2)$ active space provides a poor description of the multi-reference effects of $\mathbf{2}$ which can only be correctly described if the $\pi$-space is included thus leading to a (8,8) active space (see Ref. 58). This will lead to a stabilization of the singlet state and, by this, an increase of the singlet-triplet splitting. For the B3LYP functional, the (absolute) experimental value of $21.1 \mathrm{kcal} / \mathrm{mol}$ would be approached in this way from above. In contrast, for the BLYP functional the singlet-triplet splitting would be significantly more exaggerated than with the $(2,2)$ description (Table 2).

We conclude that the seemingly better performance of BLYP is fortuitous. A more reliable description is obtained with the B3LYP functional because in this way a double-counting of correlation effects is avoided, which can lead to an exaggeration of the stability of the singlet biradical state. It seems preferable to include multi-reference effects explicitly via ensemble DFT or BS-UDFT than implicitly via the exchange functional. This is reasonable because in the latter case long-range correlation effects enter the description in a more unspecified, not necessarily optimal way while in the former case they are fine-tuned via the active space orbitals. In the following, we have to see whether these preliminary conclusions are also valid for biradicals $\mathbf{3}$ - $\mathbf{6}$.

Biradicals 3 - 6. The biradical character of the singlet states of $\mathbf{1}$ - $\mathbf{6}$ can be assessed by the FON values of the LUMO. It increases with the value of the singlet-triplet splitting. If the latter is significantly negative (strong stabilization of the singlet state relative to the triplet state by spin-coupling), the biradical character is small while a singlet state of comparable or even higher energy than that of the corresponding triplet state possesses high biradical character. This is reflected by Figure 6 which suggests that the biradical character increases exponentially with the singlet triplet splitting in the range $-25 \leq \Delta E_{S T} \leq 5 \mathrm{kcal} / \mathrm{mol}$. The REKS and BS-UDFT $\Delta E_{S T}$ values reproduce the trend in the experimental (or CASPT2) reference value of the splittings [60-65] where in the case of $\mathbf{5}$ and $\mathbf{6}$ no exact values are known.

In the $\pi, \pi$-biradical 3 (Scheme 1), the single electrons can only interact by hyperconjugation via the $\mathrm{CH}_{2}$ group, 
which leads to little stabilization and, therefore a singlet energy slightly above the triplet energy $(0.7 \mathrm{kcal} / \mathrm{mol}$, Table 2) results. Substitution of the $\mathrm{H}$ atoms by $\mathrm{F}$ atoms (4) increases hyperconjugation due to a lowering of the pseudo- $\pi^{\star}\left(\mathrm{CF}_{2}\right)$ (or $\sigma^{\star}(\mathrm{CF})$ ) orbitals thus coupling the single electrons more effectively and stabilizing the singlet state; the biradical character is reduced from 79 to $55 \%$ (B3LYP; BLYP: from 64 to 34 \%, Table 2).

For the $\pi, \pi$-biradical 5 (Scheme 1), the situation is similar to that in 3: Now two allyl units are connected by a $\mathrm{CH}_{2}$ group so that again spin-coupling can only be mediated by hyperconjugation. Bond C4-C5 is relatively long (1.50 to $1.51 \AA$, Figure 5) indicating that $\pi$-conjugation via this bond is moderate. Steric repulsion between the exocyclic $\mathrm{CH}_{2}$-groups plays an important role in this connection (Figure 5). The singlet-triplet splitting for $\mathbf{5}$ is larger than zero. [64] Calculations indicate that it has a similar value $(0.7 \mathrm{kcal} / \mathrm{mol}$, REKS/B3LYP, Table 2) as biradical 3 because of the same coupling mechanism between the unpaired electrons.

If the $\mathrm{CH}_{2}$ group is replaced by an oxygen atom as in $\mathbf{6}$, the two allyl units can conjugate via the $\pi$ orbital of the heteroatom thus leading to an effective coupling of the single electrons. The singlet state is stabilized below the triplet state and the singlet-triplet splitting becomes $-2.5 \mathrm{kcal} / \mathrm{mol}$ (REKS/B3LYP/cc-pVTZ, Table 2). This is in agreement with the experimental observation that the singlet-triplet splitting is negative for 6 . [66]

The data in Table 2 together with the information provided by Figure 6 confirm what was discussed in the case of the benzyne biradicals. The hybrid functionals always lead to a stronger explicit account of multi-reference effects, which is clearly due to the fact that the self-interaction error is reduced by the admixture of exact exchange and, by this, also the long-range correlation effects simulated by the exchange functional. Accordingly, a larger biradical character is predicted for the singlet states $\mathbf{1}$ - 6 (Table 2, Figure 6).

The question whether implicit or explicit account of multi-reference effects leads to a better description can be answered by considering the calculated singlet-triplet splittings. Due to additional implicit account of electron correlation effects via the exchange functional, BLYP stabilizes the singlet state stronger than B3LYP thereby increasing absolute splittings $\left(\Delta E_{S T}<0\right)$ beyond or reducing them $\left(\Delta E_{S T}>0\right)$ below the experimental value (Table 2).

The implicit account of multi-reference effects is the more economic way to describe biradicals and other type II systems. However, an unspecified coverage of these effects as provided by the presently available approximate exchange functionals cannot guarantee sufficient accuracy. Hence, one has to explicitly add multi-reference effects to get the correct state ordering and accurate relative energies. An explicit addition of multi-reference effects, however, will only be efficient if implicit coverage of multi-reference effects via the exchange functional is largely suppressed, i.e. if one works with partially (or fully) self-interaction corrected functionals such as the hybrid functionals. Hence, B3LYP rather than BLYP has to be used when BS-UDFT, REKS or other multi-configurational methods should be applied in an efficient and economic way.

But even in the case of a hybrid functional such as B3LYP, one cannot exclude a double counting of electron correlation effects. This double counting refers to dynamic rather than non-dynamic electron correlation effects as was discussed above. The variational nature of the REKS orbitals and orbital occupation numbers enables one to separate the non-dynamic correlation effects included explicitly and those included implicitly via the exchange functional. Thus, the double counting of the non-dynamic correlation energy is largely avoided by REKS. The same can not be guaranteed for the dynamic correlation energy. While for the two limiting cases, 'normal' closed-shell system and open-shell singlet system, REKS reproduces the conventional KS results ( $i$. e., no double counting), the description of intermediate situations depends on the approximations made in the REKS energy expression 


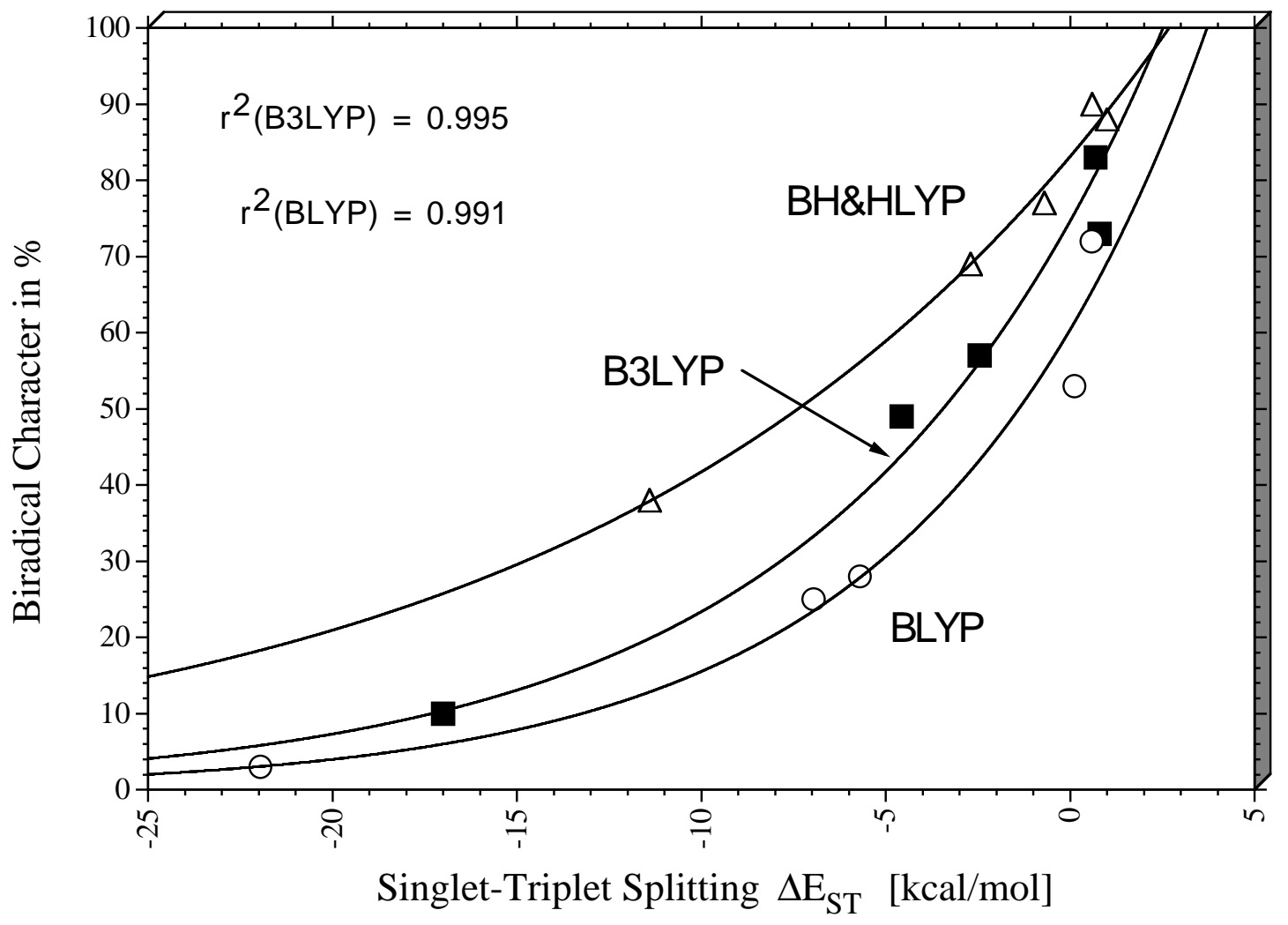

Figure 6: The biradical character of molecules $\mathbf{1}-\mathbf{5}$ in their lowest singlet state calculated as $100 \times$ FON(LUMO). $\operatorname{REKS}(2,2) / 6-31 \mathrm{G}(\mathrm{d})$ calculations. 
(Eq. 14). Hopefully, the extent of an extra dynamic correlation energy covered by the $2 \times 2$ secular problem in REKS is mitigated by the inclusion of the dynamic correlation terms into the coupling term $D\left(\phi_{r}, \phi_{s}\right)$ of Eq. (14). However, a more precise knowledge is necessary of the dependence of the weighting factor in front of the coupling term $D\left(\phi_{r}, \phi_{s}\right)$ of Eq. (14) on the fractional occupation numbers of orbitals to avoid precisely the double counting of the dynamic correlation in REKS.

\section{Explicit Coverage of Multi-reference Effects: Merging of DFT and WFT}

The problem of an accurate or at least a reasonable description of a multi-reference system has been satisfactorily solved for WFT using multi-configurational techniques. [67] Therefore, it is attractive to combine a correct multiconfigurational description of non-dynamic electron correlation effects with the DFT coverage of dynamic electron correlation effects. Various steps in this direction have been made and a review on the topic was given by one of us. [13] Therefore, we summarize here only the essentials of the CAS(complete active space)-DFT method [32] while other approaches to the problem can be found in the literature [13] (for some recent references, see [68-70]).

In view of the discussions in section 2, the combination of WFT and DFT methods must imply that the exchange part is calculated exactly at the WFT level and that a self-interaction free correlation functional such as the LYP [45] or the Colle-Salvetti functional [49] is used. Considering these two aspects, CAS-DFT was derived by Gräfenstein and Cremer [32] utilizing results of Miehlich and co-workers for the homogeneous electron gas. [71]

For CAS-DFT, the standard Kohn-Sham approach is changed in the way that the minimization procedure is done over all properly antisymmetric CAS wave functions for a given size of the active space. Also, the Hartree energy $E_{J}$ is replaced by the full electron interaction energy $V_{e e}$ of the CAS trial wave function, which covers exact exchange and the non-dynamic electron correlation effects. Hence, the CAS-DFT functional is given by Eq. (15):

$$
F^{\mathrm{CAS}-\mathrm{DFT}}[\rho]=\min _{\Psi \mathrm{CAS} \rightarrow \rho}\left\langle\Psi^{\mathrm{CAS}}\left|\hat{T}+\hat{V}_{e e}\right| \Psi^{\mathrm{CAS}}\right\rangle
$$

Adding to $(15)$ the dynamic correlation energy $E_{\mathrm{C}}^{\mathrm{CAS}-\mathrm{DFT}}[\rho]$ as determined with a suitable correlation functional for the CAS density leads to Eq. (16):

$$
F[\rho]=F^{\mathrm{CAS}-\mathrm{DFT}}[\rho]+E_{\mathrm{C}}^{\mathrm{CAS}-\mathrm{DFT}}[\rho],
$$

Of course, one cannot use spin densities $\rho_{\alpha}$ and $\rho_{\beta}$ to determine $F[\rho]$ because they lead to errors in the description of state multiplets. Therefore, these densities are replaced by the total CAS density $\rho(\mathbf{r})$ and the CAS on-top pair density $P(\mathbf{r}, \mathbf{r})$ (the second electron is on-top of the reference electron thus leading to a simplified pair density distribution) as input quantities for the correlation functional [32] as was originally suggested by Moscardo and SanFabian. [72] In this way, differences between states with different multiplicity can be distinguished. By employing the Colle-Salvetti functional [49], which uses the total density and pair density directly as input quantities, there is no need for a conversion of the functional as would be the case for any other gradient-corrected correlation functional based on spin densities $\rho_{\alpha}$ and $\rho_{\beta}$.

Although the construction of a suitable CAS-DFT method seems to be straightforward, its realization is hampered by the fact that the CASSCF description includes beside non-dynamic electron correlation also an unknown amount of dynamic correlation effects and, accordingly, the functional $F[\rho]$ of Eq. (16) leads to some doublecounting of short-range correlation effects. A way out of this dilemma is to estimate the amount of dynamic 
electron correlation covered by the CASSCF description utilizing the homogeneous electron gas as an appropriate reference system. $[32,68]$ A reference density $\rho_{\text {ref }}(\mathbf{r})$ is determined by considering all core orbitals and active space orbitals to be doubly occupied so that the magnitude of $\rho_{\text {ref }}(\mathbf{r})$ reflects the size of the active space. If compared with the true density of the system in question, $\rho(\mathbf{r})$, the ratio $\rho_{\text {ref }}(\mathbf{r}) / \rho(\mathbf{r}) \geq 1$ will increase with the size of the active space as will the amount of dynamic electron correlation already covered by the CASSCF wave function. Hence, one has to scale the DFT correlation energy locally by a scaling factor $f(0 \leq f \leq 1)$ to avoid this double counting.

For this purpose, one calculates the correlation energy of the homogeneous electron gas (HEG) with the density $\rho$ in a way that is comparable to the CASSCF description: Excitations are allowed from the occupied orbitals only into virtual orbitals up to a certain energy limit, which is adjusted in the way that the active space has just the reference density $\rho_{\text {ref }}$. For the determination of the scaling factor $\mathrm{f}$, the resulting correlation energy $\varepsilon_{C}^{\mathrm{HEG}}\left(\rho, \rho_{\text {ref }}\right)$ is related to the value $\varepsilon_{C}^{\mathrm{HEG}}(\rho, \infty)$ for all virtual orbitals being included. [32] The correlation energy is defined by Eq. (17).

$$
E_{C}^{\mathrm{CAS}-\mathrm{DFT}}=\left.\int \mathrm{d}^{3} r \quad f\left(\rho, \rho_{\mathrm{ref}}\right) \varepsilon_{C}(\rho, \nabla \ldots, P, \nabla P, \ldots)\right|_{\mathbf{r}} .
$$

where the correlation energy per electron, $\varepsilon_{C}$ depends on the total and the on-top pair density as well as on their gradients (apart from higher terms in the most general case).

Although the scaling procedure eliminates the most serious errors due to a double-counting of dynamic electron correlation, the uniform treatment of core and active space orbitals can still jeopardize a successful application of CAS-DFT. For example, the core part of a CASSCF description of a chemical reaction may change, which can only be covered if core and active space orbitals are explicitly distinguished in the scaling procedure based on the homogeneous electron gas. [73] Also, one has to differentiate between singly and doubly occupied orbitals in the scaling procedure if one wants to get reliable energy differences between ground and excited states having different orbital occupancies. For example in the case of a singlet-triplet energy difference a scaling procedure not differentiating between singly and doubly occupied orbitals will predict the triplet stability too small thus underestimating $\left(\Delta E_{S T}>0\right)$ or overestimating $\left(\Delta E_{S T}<0\right)$ the singlet-triplet splitting because of an exaggerated down-scaling of dynamic electron correlation in the triplet. [73] However, once these problems are solved, CAS-DFT can provide a reliable and computationally feasible account of both non-dynamic and dynamic electron correlation effects.

\section{Conclusions}

This work has shown that a double-counting of electron correlation effects is a serious problem that accompanies extensions of Kohn-Sham DFT to some form of multi-reference DFT. Double-counting can occur for non-dynamic, dynamic or both types of electron correlation depending on the method used. It is a reflection of the fact that electron correlation is covered by DFT implicitly via the form of the exchange-correlation functional and any explicit additional coverage of correlation effects is difficult to adjust in a way that any double-counting correlation is avoided. Implicit coverage of correlation effects as given by currently available approximate exchange-correlation functionals cannot be correctly understood without considering the self-interaction error of these functionals. In the exchange part, the self-interaction error leads to the simulation of long-range correlation effects provided restricted Kohn-Sham DFT is used. Exact exchange obtained with restricted theory is delocalized over the interacting 
centers while DFT exchange is localized. If the distance between the interacting centers in a multi-reference system increases (dissociating molecules, biradicals, etc.) the self-interaction error and the non-dynamic correlation effects mimicked also increase due to stronger delocalization of the exact exchange hole. In the case of an unrestricted theory the exact exchange hole is localized as is the UDFT exchange hole. In this situation, both long-range and short-range correlation effects can be mimicked where with increasing distance between the interacting centers the latter effects dominate.

In this work, we have not considered the consequences of the self-interaction error of correlation functionals such as VWN5 or PW91 because this is significantly smaller than the error in the exchange functional and partially canceled by the latter. It has been shown that the self-interaction error of the correlation functional decreases the correlation energy (values become more negative), strengthens the chemical bond, and thereby the stability of the molecule is increased. [14] Accordingly, it represents another, although much smaller factor when analyzing the implicit account of electron correlation.

Depending on what method is used to explicitly account for multi-reference effects double-counting of electron correlation may lead to different situations. The REKS method carried out with a small active space should add only non-dynamic electron correlation. We have given arguments that state mixing will be sensitive to the longrange effects introduced by the exchange functional thus minimizing a double-counting of non-dynamic electron correlation. However, REKS, BS-UDFT and other multi-configurational DFT methods will lead to a doublecounting of dynamic electron correlation due to an implicit introduction of these effects by the self-interaction error of the exchange functional. There is no guarantee that ensemble DFT or any multi-configurational DFT can correct this double-counting automatically.

There are two escape routes out of this situation: a) Exchange functionals can be used that do not or do only partially suffer from the self-interaction error. The hybrid exchange functionals represent a possible although not perfect alternative in this connection. Consequently, BS-UDFT or REKS calculations should be carried out with a hybrid functional such as B3LYP rather than a GGA functional such as BLYP.

b) Exchange is calculated correctly within WFT using methods such as GVB-DFT, [31] MC-DFT or CAS-DFT. [32] In this way, a double-counting of multi-reference effects is excluded. As long as a two-configurational description is sufficient to cover explicitly multi-reference effects, also the problem of double-counting dynamic electron correlation effects becomes negligible. However, with an increase of the active space the WFT method in question may introduce beside non-dynamic electron correlation effects also unwanted dynamic electron correlation effects already covered by the correlation functional. In this case there are however scaling methods available that largely reduce the double-counting of dynamic electron correlation.

Although a primary goal of current research in DFT is to preserve its simplicity while at the same time extending its applicability, there are molecular systems for which a correct DFT description can only be obtained by replacing Kohn-Sham DFT by the more costly ensemble DFT. A routine application of ensemble DFT is presently not possible and therefore requires further work on this topic. In this connection, it will be an important task to exactly understand the role of the self-interaction error and to consider new alternatives of its systematic reduction. 


\section{Acknowledgment}

At Göteborg University, this work was supported by the Swedish Natural Science Research Council (NFR). Calculations were done on the supercomputers of the Nationellt Superdatorcentrum (NSC), Linköping, Sweden. The authors thank the NSC for a generous allotment of computer time. The work at Hebrew University was supported in part by the Niedersachsen Foundation, the German Israeli Foundation (GIF), and the Robert Szold Fund.

\section{References}

[1] (a) Hohenberg, P.; Kohn, W. Inhomogeneous Electron Gas. Phys. Rev. 1964, 136, B 864-871. (b) Kohn, W.; Sham, L. J. Self-Consistent Equations Including Exchange and Correlation Effects. Phys. Rev. 1965, 140, A $1133-1138$.

[2] Parr, R. G.; Yang, W. in International Series of Monographs on Chemistry 16: Density-Functional Theory of Atoms and Molecules; Oxford University Press: New York, 1989.

[3] A Chemist's Guide to Density Functional Theory, Koch W.; Holthausen M. C., Wiley-VCH: New York, 2001.

[4] For reviews on DFT methods, see for example (a) Density Functional Methods in Chemistry, Labanowski, J. K.; Andzelm, J. W., Eds; Springer: Heidelberg, 1990. (b) Theoretical and Computational Chemistry, Vol. 2, Modern Density Functional Theory - A Tool For Chemistry, Seminario, J. M.; Politzer, P., Eds.; Elsevier: Amsterdam, 1995. (c) Chemical Applications of Density Functional Theory, ACS Symposium Series 629, Laird, B. B.; Ross, R. B.; Ziegler, T., Eds; American Chemical Society: Washington, DC, 1996. (d) Lecture Notes in Physics, Density Functionals: Theory and Applications , Joubert, D., Ed.; Springer: Heidelberg, 1997. (e) Recent Advances in Computational Chemistry, Vol. 1, Recent Advances in Density Functional Methods, Part II, Chong, D. P., Ed.; World Scientific: Singapore, 1997. (f) Electronic Density Functional Theory, Recent Progress and New Directions, Dobson, J. F.; Vignale, G.; Das, M. P., Eds.; Plenum Press: New York, 1998. (g) Gill, P. Density Functional Theory (DFT), Hartree-Fock (HF), and the Self-Consistent Field, in Encyclopedia of Computational Chemistry, Schleyer, P. v. R.; Allinger, N. L.; Clark, T.; Gasteiger, J.; Kollman, P. A.; Schaefer III, H. F.; Schreiner, P. R., Eds., Wiley: Chichester, UK, 1998, Vol. 1, p. $678-689$.

[5] (a) Schipper, P. R. T.; Gritsenko, O. V.; Baerends, E. J. One-determinantal pure state versus ensemble Kohn-Sham solutions in the case of strong electron correlation: $\mathrm{CH}_{2}$ and $\mathrm{C}_{2}$ Theor. Chem. Acc. 1998, 99, 329-343. (b) Schipper, P. R. T.; Gritsenko, O. V.; Baerends, E. J. Benchmark calculations of chemical reactions in density functional theory: Comparison of the accurate KohnSham solution with generalized gradient approximations for the $H_{2}+H$ and $H_{2}+H_{2}$ reactions J. Chem. Phys. 1999, 111, 4056-4067.

[6] (a) Savin, A. Beyond the Kohn-Sham Determinant, in Recent Advances in Density Functional Methods, Part I, Chong, D. P., Ed.; World Scientific: Singapore, 1995, p. 123-153. (b) Savin, A. On Degeneracy, Near Degeneracy and Density Functional Theory, in Recent Developments of Modern Density Functional Theory, Seminario, J. M., Ed; Elsevier, Amsterdam, 1996, 327-357.

[7] Ziegler, T.; Rauk, A.; Baerends, E. J. On the calculation of multiplet energies by the Hartree-Fock-Slater method. Theor. Chim. Acta 1977, 43, 261-271. 
[8] von Barth, U. Local-density theory of multiplet structure Phys. Rev. A 1979, 20, 1693-1703.

[9] Gräfenstein, J.; Kraka, E.; Filatov, M.; Cremer, D. Can Unrestricted Density-Functional Theory Describe Open Shell Singlet Biradicals? Int. J. Mol. Science, in press.

[10] It has been pointed out that this statement is only correct when including ensemble DFT. See, Ref.s 5,27 , and 28 .

[11] Becke, A. D., Exchange-Correlation Approximations in Density-Functional Theory, in Advanced Series in Physical Chemistry, Vol. 2, Modern Electronic Structure Theory, Part II, Yarkony, D. R., Ed; World Scientific: Singapore,1995, p. 1022-1046;

[12] a) Gritsenko, O. V.; Schipper, P. R. T.; Baerends, E. J. Exchange and correlation energy in density functional theory: Comparison of accurate density functional theory quantities with traditional Hartree-Fock based ones and generalized gradient approximations for the molecules $L i_{2}, N_{2}, F_{2}$. J. Chem. Phys. 1997, 107, 50075015 . b) Schipper, P. R. T.; Gritsenko, O. V.; Baerends, E. J. Kohn-Sham potentials and exchange and correlation energy densities from one- and two-electron density matrices for $\mathrm{Li}_{2}, \mathrm{~N}_{2}, F_{2}$. Phys. Rev. A, 1998, 57, 1729-1742. c) Gritsenko, O. V.; Ensing, B.; Schipper, P. R. T.; Baerends, E. J. Comparison of the Accurate Kohn-Sham Solution with the Generalized Gradient Approximations (GGAs) for the SN2 Reaction $\mathrm{F}^{-}+\mathrm{CH}_{3} \mathrm{~F} \rightarrow \mathrm{FCH}_{3}+\mathrm{F}^{-}:$A Qualitative Rule To Predict Success or Failure of GGAs J. Phys. Chem. A, 2000, 104, 8558-8565. d) Handy, N. C.; Cohen, A. J. Left-right correlation energy Mol. Phys., 2001, 99, $403-413$.

[13] Cremer, D.; Density Functional Theory - Coverage of Dynamic and Nondynamic Electron Correlation Effects Mol. Phys. 2001, 000-000.

[14] (a) Polo, V.; Kraka, E.; Cremer, D. Nondynamic Electron Correlation and the Self-Interaction Error of Density Functional Theory Mol. Phys. (Davidson issue), 2002 in press.

(b) He, Y.; Gräfenstein, J.; Kraka, E. ; Cremer, D. What Correlation Effects Are Covered by Density Functional Theory? Mol. Phys. 2000, 98, 1639-1658.

[15] Polo, V.; Gräfenstein, J.; Kraka, E.; Cremer, D. Influence of the Self-Interaction Error on the Structure of the DFT Exchange Hole Chem. Phys. Lett., 2002, 352, 469-478.

[16] Polo, V.; Kraka, E.; Cremer, D. Some Thoughts about the Stability and Reliability of Commonly Used Exchange-Correlation Functionals - Coverage of Dynamic and Nondynamic Correlation Effects Theoret. Chem. Accounts, 2002, in press.

[17] Perdew, J.P.; Zunger, A. Self-interaction correction to density-functional approximations for many-electron systems. Phys. Rev. B 1981, 23, 5048-5079.

[18] a) Perdew, J.P. In Local Density Approximations in Quantum Chemistry and Solid State Physics; Avery, J., Dahl, J.P., Eds.; New York: Plenum Press, 1984.

b) Perdew, J.P.; Ernzerhof, M. Driving out the self-interaction error. In Electronic Density Functional Theory: Recent Progress and New Directions; Dobson, J.F., Vignale, G., Das, M.P., Eds.; New York: Plenum Press, 1998; p. 31-41. 
[19] Harrison, J.G.; Heaton, R.A.; Lin, C.C. Self-interaction correction to the local density Hartree-Fock atomic calculations of excited and ground states. J. Phys. B: At. Mol. Phys. 1983, 16, 2079-2092.

[20] a) Pederson, M.R.; Heaton, R.A.; Lin, C.C. Local-density Hartree-Fock theory of electronic states of molecules with self-interaction correction. J. Chem. Phys. 1984, 80, 1972-1982.

b) Pederson, M.R.; Heaton, R.A.; Lin, C.C. Density-functional theory with self-interaction correction: Application to the lithium molecule. J. Chem. Phys 1985, 82, 2688-2699.

[21] Svane, A.; Temmerman, W.M.; Szotek, Z.; Laegsgaard, J.; Winter, H. Self-interaction-corrected local-spindensity calculations for rare earth materials. Int. J. Quantum Chem. 1999, 77, 799-813.

[22] Goedecker, S.; Umrigar, C.J. Critical assessment of the self-interaction-corrected-local-density-functional method and its algorithmic implementation. Phys. Rev. A 1977, 55, 1765-1771.

[23] a) Li, Y.; Krieger, J.B. Construction and application of gradient-expansion approximations for exchange energy with self-interaction correction. Phys. Rew. A 1990, 41, 1701-1704;

b) Chen, J.; Krieger, J.B.; Li, Y. Kohn-Sham calculations with self-interaction-corrected local-spin-density exchange-correlation energy functional for atomic systems. Phys. Rev. A 1996, 54, 3939-3947.

[24] a) Garza, J.; Nichols, J.A.; Dixon, D.A. The optimized effective potential and the self-interaction correction in density functional theory: Application to molecules. J. Chem. Phys. 2000 112, 7880-7890.

b) Garza, J.; Nichols, J.A.; Dixon, D.A. The role of the local-multiplicative Kohn-Sham potential on the description of occupied and unoccupied orbitals. J. Chem. Phys. 2000, 113, 6029-6033.

c) Garza, J.; Vargas, R.; Nichols, J.A.; Dixon, D.A. Orbital energy analysis with respect to LDA and selfinteraction corrected exchange-only potentials. J. Chem. Phys. 2001, 114, 639-651.

[25] a) Guo, Y.; Whitehead, M.A. An alternative Self-interaction correction in the generalized exchange localdensity functional theory. J. Comp. Chem. 1991, 12, 803-810.

b) Whitehead, M.A.; Suba, S. In Recent Advances in Computational Chemistry - Vol.1, Recent Advances in Density Functional Methods, Part I; Chong, D.P., Ed.; Singapore: World Scientific, 1995; p. 53-78.

[26] Gill P.M.W.; Johnson B.G.; Gonzales C.A.; Pople J.A. A density functional study of the simplest hydrogen abstraction reaction. Effect of self-interaction correction. Chem. Phys. Lett. 1994, 221, 100-108.

[27] Lieb, E. H. Density Functionals for Coulomb Systems. Int. J. Quantum Chem. 1983, 24, $243-277$.

[28] a) Englisch, H.; Englisch, R. Exact Density Functionals for Ground-State Densities. I. General Results Phys. Stat. Sol (b) 1984, 123, 711-721. b) Englisch, H.; Englisch, R. Exact Density Functionals for Ground-State Densities. II. Details and Remarks Phys. Stat. Sol (b) 1984, 124, 373-379. c) Levy, M. Electron densities in search of Hamiltonians Phys. Rev. A 1982, 26, 1200-1208.

[29] a) Filatov, M.; Shaik, S. A spin-restricted ensemble-referenced Kohn-Sham method and its application to diradicaloid situations. Chem. Phys. Lett. 1999, 304, 429-437; b) Filatov, M.; Shaik, S. Artificial symmetry breaking in radicals is avoided by the use of the Ensemble-Referenced Kohn-Sham (REKS) method Chem. Phys. Lett. 2000, 332, 409-419. 
[30] a) Filatov, M.; Shaik, S. Tetramethyleneethane (TME) Diradical: Experiment and Density Functional Theory Reach an Agreement J. Phys. Chem. A 1999, 103, 8885-8889; b) Filatov, M.; Shaik, S.; Woeller, M.; Grimme, S; Peyerimhoff, S. Locked alkenes with a short triplet state lifetime Chem. Phys. Lett. 2000, 316, 135-140; c) Filatov, M.; Shaik, S. Diradicaloids: Description by the Spin-Restricted Ensemble-Referenced Kohn-Sham Density Functional Method J. Phys. Chem. A 2000, 104, 6628-6636; d) Filatov, M.; Shaik, S.; de Visser, S. P. REKS calculations on ortho-, meta- and para-benzyne Phys. Chem. Chem. Phys. 2000, 2, 5046-5048; e) Filatov, M.; Shaik, S.; de Visser, S. P. Myers-Saito and Schmittel cyclization of hepta-1,2,4-triene-6-yne: A theoretical REKS study Phys. Chem. Chem. Phys. 2001, 3, 1242-1245.

[31] (a) Kraka, E. Homolytic dissociation-energies from GVB-LSDC calculations. Chem. Phys. 1992, 161, 149153. (b) Kraka, E.; Cremer, D.; Nordholm, S.A New Way of Calculating Bond Dissociation Energies, in Molecules in Natural Science and Biomedicine; Maksic, Z., Eckert-Maksic, M., Eds.; Ellis Horwood, Chichester, 1991; p. 351-374. (c) In the case of the OSS state of $\mathbf{1}$ or $\mathbf{2}$, the GVB-DFT description simplifies to a ROHF-DFT description. See, Davidson, E. R. Spin-restricted open-shell self-consistent-field theory. Chem. Phys. Lett. 1973, 21, 565-567.

[32] (a) Gräfenstein, J; Cremer, D. The combination of density functional theory with multi-configuration methods - CAS-DFT. Chem. Phys. Lett. 2000, 316, 569-577.

(b) Gräfenstein, J.; Cremer, D. Can density functional theory describe multi-reference systems? Investigation of carbenes and organic biradicals. Phys. Chem. Chem. Phys. 2000, 2, 2091-2103.

[33] Gräfenstein, J.; Cremer, D.; Kraka, E. Density functional theory for open-shell singlet biradicals. Chem. Phys. Lett. 1998, 288, 593-602.

[34] a) Filatov, M.; Shaik, S. Spin-restricted density functional approach to the open-shell problem. Chem. Phys. Lett. 1998, 288, 689-697; b) Filatov, M.; Shaik, S. Application of spin-restricted open-shell Kohn-Sham method to atomic and molecular multiplet states. J. Chem. Phys. 1999, 110, 116-125.

[35] Dunning Jr., T. H. Gaussian-basis sets for use in correlated molecular calculations. 1. The atoms boron through neon and hydrogen. J. Chem. Phys. 1989, 90, 1007-1023.

[36] Slater, J. C. A Simplification of the Hartree-Fock Method Phys. Rev. 1951, 81, 385-390.

[37] Vosko, S. H.; Wilk, L.; Nusair, M. Accurate spin-dependent electron liquid correlation energies for local spin density calculations: a critical analysis Can J. Phys. 1980, 58, 1200-1211.

[38] Polo, V.; Kraka, E.; Cremer, D., to be published.

[39] See, e.g. , Baerends, E. J.; Gritsenko, O. V. A Quantum Chemical View of Density Functional Theory. J. Phys. Chem. A, 1997, 101, 5383-5403.

[40] Note that there is also a difference between VWN5 correlation and SIC-VWN5 correlation, $[14 \mathrm{~b}, 15,16]$ which is not considered in this work. 
[41] (a) Langreth, D. C.; Perdew, J. P. The exchange correlation energy of a metallic surface. Solid State Commun. 1975, 17, 1425-1429. (b) Gunnarsson, O.; Lundqvist, B. I. Exchange and correlation in atoms, molecules, and solids by the spin-density-functional formalism. Phys. Rev. B 1976, 13, 4274-98.

[42] Harris, J. Adiabatic connection approach to Kohn-Sham theory Phys. Rev. A 1998, 288, 689-697.

[43] Hariharan, P. C.; Pople, J. A. The Influence of Polarization Functions on Molecular Orbital Hydrogenation Energies. Theor. Chim. Acta 1973, 28, 213-222.

[44] Becke, A. D. Density-functional exchange-energy approximation with correct asymptotic-behavior. Phys. Rev. A 1988, 38, 3098-3100.

[45] Lee, C.; Yang, W.; Parr, R. P. Development of the Colle-Salvetti correlation-energy formula into a functional of the electron-density. Phys. Rev. B 1988, 37, 785-789.

[46] (a) Becke, A. D. Density-functional thermochemistry. 3. The role of exact exchange. J. Chem. Phys. 1993, 98, 5648-5652. See also (b) Stephens, P. J.; Devlin, F. J.; Chabalowski, C. F.; Frisch, M. J. Ab-initio calculation of vibrational absorption and circular-dichroism spectra using density-functional force-fields. J. Phys. Chem. 1994, 98, 11623-11627.

[47] Becke, A. D. A new mixing of HartreeFock and local density-functional theories J. Chem. Phys. 1993, 98, $1372-1377$.

[48] a) Perdew, J. P., in Electronic Structure of Solids '91, Ziesche, P.; Eschrig, H., Eds. Akademie-Verlag: Berlin, 1991, p. 11. b) Perdew, J.P. and Wang, Y., Accurate and simple analytic representation of the electron-gas correlation energy. Phys. Rev. B 1992, 45, 13244-13249.

[49] a) Colle, R.; Salvetti, O. Approximate calculation of the correlation energy for the closed shells Theor. Chim. Acta 1975, 37, 329-334. b) Colle, R.; Salvetti, O. Generalization of the Colle-Salvetti correlation-energy method to a many-determinant wave-function J. Chem. Phys. 1990, 93, 534-544.

[50] Kraka, E.; Gräfenstein, J.; Gauss, J.; Filatov, M.; Polo, V.; Reichel, F.; Olsson, L.; Konkoli, Z; He, Z; Cremer, D. COLOGNE2001, Göteborg University, Göteborg, 1999.

[51] Frisch, M. J.; Trucks, G. W.; Schlegel, H. B.; Scuseria, G. E.; Robb, M. A.; Cheeseman, J. R.; Zakrzewski, V. G.; Montgomery, Jr., J. A.; Stratmann, R. E.; Burant, J. C.; Dapprich, S.; Millam, J. M.; Daniels, A. D.; Kudin, K. N.; Strain, M. C.; Farkas, O.; Tomasi, J.; Barone, V.; Cossi, M.; Cammi, R.; Mennucci, B.; Pomelli, C.; Adamo, C.; Clifford, S.; Ochterski, J.; Petersson, G. A.; Ayala, P. Y.; Cui, Q.; Morokuma, K.; Malick, D. K.; Rabuck, A. D.; Raghavachari, K.; Foresman, J. B.; Cioslowski, J.; Ortiz, J. V.; Stefanov, B. B.; Liu, G.; Liashenko, A.; Piskorz, P.; Komaromi, I.; Gomperts, R.; Martin, R. L.; Fox, D. J.; Keith, T.; Al-Laham, M. A.; Peng, C. Y.; Nanayakkara, A.; Gonzalez, C.; Challacombe, M.; Gill, P. M. W.; Johnson, B.; Chen, W.; Wong, M. W.; Andres, J. L.; Gonzalez, C.; Head-Gordon, M.; Replogle, E. S.; Pople, J. A. Gaussian 98, Revision A.5, Gaussian, Inc., Pittsburgh PA, 1998. 
[52] (a) Kraka, E.; Cremer, D. CCSD(T) investigation of the Bergman cyclization of enediyne - Relative stability of $o$-didehydrobenzene, $m$-didehydrobenzene, and $p$-didehydrobenzene. J. Am. Chem. Soc. 1994, 116, 49294936. (b) Kraka, E.; Cremer, D. Ortho-benzyne, meta-benzyne, and para-benzyne - A comparative CCSD(T) investigation. Chem. Phys. Lett. 1993, 216, 333-340.

[53] Gräfenstein, J.; Hjerpe, A. M.; Kraka, E.; Cremer, D. An accurate description of the Bergman reaction using restricted and unrestricted DFT: Stability test, spin density, and on-top pair density. J. Phys. Chem. A, 2000, 104, 1748-1761.

[54] Kraka, E.; Cremer, D. The para-didehydropyridine, para-didehydropyridinium, and related biradicals - A contribution to the chemistry of enediyne antitumor drugs. J. Comp. Chem. 2001, 22, 216-229.

[55] Kraka, E.; Cremer, D. Computer design of anticancer drugs. A new enediyne warhead. J. Am. Chem. Soc. 2000, 122, 8245-8264.

[56] Crawford, T. D.; Kraka, E.; Stanton, J. F.; Cremer, D. Problematic p-Benzyne: Orbital Instabilities, Biradical Character, and Broken Spin Symmetry. J. Chem. Phys. 2001, 114, 10638-10650.

[57] Kraka, E.; Cremer, D.; Bucher, G.; Wandel, H.; Sander, W. A CCSD(T) and DFT investigation of $m$-benzyne and 4-hydroxy-m-benzyne. Chem. Phys. Lett. 1997, 268, 313-320.

[58] Kraka,E.; Anglada, J.; Hjerpe, A.; Filatov, M.; Cremer, D. m-Benzyne and Bicyclo[3.1.0]hexatriene - Which Isomer is more Stable? - A Quantum Chemical Investigation. Chem. Phys. Lett. 2001, 348, 115-125.

[59] Winkler, M.; Sander, W. The Structure of meta-Benzyne Revised - A Close Look into $\sigma$-Bond Formation. J. Phys. Chem. A 2001, 105, 10422-10432.

[60] Wenthold, P. G.; Squires, R. R.; Lineberger, W. C. Ultraviolet Photoelectron Spectroscopy of the $o^{-}, m^{-}$, and $p$ - Benzyne Negative Ions. Electron Affinities and Singlet-Triplet Splittings for $o_{-}, m^{-}$, and $p$ - Benzyne. J. Am. Chem. Soc. 1998, 120, 5279-5290.

[61] a) Wierschke, S. G.; Nash, J. J; A multiconfigurational SCF and correlation-consistent CI study of the structures, stabilities, and singlet-triplet splittings of $o$-benzyne, $m$-benzyne, and $p$-benzyne. Squires, R. R., J. Am. Chem. Soc. 1993, 115, 11958-11967. b) Wenthold, P. G.; Paulino, J. A.; Squires, R. R. The absolute heats of formation of ortho-benzyne, meta-benzyne, and para-benzyne. J. Am. Chem. Soc. 1991, 113, 7414-7415. c) Wenthold, P. G.; Squires, R. R. Biradical thermochemistry from collision-induced dissociation threshold energy measurements - Absolute heats of formation of ortho-benzyne, meta-benzyne, and para-benzyne. J. Am. Chem. Soc. 1994, 116, 6401-6412.

[62] Skancke, A.; Hrovat, D. A.; Borden W. T. Ab initio calculations of the effects of geminal silyl substituents on the stereomutation of cyclopropane and on the singlet-triplet splitting in trimethylene. J. Am. Chem. Soc., 1998, 120, 7079-7084.

[63] For a recent review, see Bally, T.; Borden, W. T. Calculations on Open Shell Molecules: A Beginner's Guide, in Reviews Comp. Chem. Lipkowitz, K. B.; Boyd, D. B., Eds.; Wiley: New York, 1999; Vol. 13, p. 1-97. 
[64] Roth, W. R.; Kowalczik, U.; Maier, G.; Reisenauer, H. P.; Sustmann, R.; Müller, W. 2,2-dimethyl-4,5dimethylene-1,3-cyclopentanediyl Angew. Chem. Int. Ed. Engl. 1987, 26, 1285-1287.

[65] Nash, J. J.; Dowd, P.; Jordan, K. D. Theoretical study of the low-lying triplet and singlet states of diradicals: prediction of ground-state multiplicities in cyclic analogues of tetramethyleneethane J. Am. Chem. Soc. 1992, 114, 10071-10072.

[66] Zilm, K. W.; Merill, R. A.; Greenberg, M. M.; Berson, J. A. The first magic angle spinning NMR spectrum of a captive intermediate: Direct observation of a singlet ground state biradical, 3,4-dimethylenefuran J. Am. Chem. Soc. 1987, 109, 1567-1569.

[67] See, e.g., a) Werner, H.-J. Matrix-Formulated Direct Multiconfiguration Self-Consistent Field and Multiconfiguration Reference Configuration-Interaction Methods in sl Advances in Chemical Physics, Volume LXIX, Ab Initio Methods in Quantum Chemistry, Part II, Lawlay, K. P., Ed.; Wiley: New York, 1987, p. 1-62. b) Shepard, R. The Multiconfiguration Self-Consistent Field Method, in sl Advances in Chemical Physics, Volume LXIX, Ab Initio Methods in Quantum Chemistry, Part II, Lawlay, K. P., Ed.; Wiley: New York, 1987, p. 63-200. c) Szalay, P. G. Towards State-Specific Formulation of Multireference Coupled-Cluster Theory, in Modern Ideas in Coupled-Cluster Methods, Bartlett, R. J. Ed.; World Scientific: Singapore, 1997; p. 81-123.

[68] Borowski, P.; Jordan, K. D.; Nichols, J.; Nachtigall P. Investigation of a hybrid TCSCF-DFT procedure Theor. Chem. Acc. 1998, 99, 135-140.

[69] Grimme, S.; Waletzke, M. A combination of Kohn Sham density functional theory and multi-reference configuration interaction methods J. Phys. Chem. 1999, 111, 5645-5655.

[70] Pollet, R.; Savin, A.; Leininger, T.; Stoll, H. Combining multideterminantal wave functions with density functionals to handle near-degeneracy in atoms and molecules J. Phys. Chem. 2002, 116, 1250-1258.

[71] a) Miehlich, B.; Stoll, H.; Savin, A. A correlation-energy density functional for multideterminantal wavefunctions. Mol. Phys., 1997, 3, 527-536. b) Savin, A. Int. J. Quant. Chem. Symp. 1988, 22, 59.

[72] Moscardò, F.; SanFabiàn E. Density-functional formalism and the two-body problem Phys. Rev. 1991, A 44, 1549-1553.

[73] Gräfenstein, J.; Cremer, D., to be published. 